\title{
On sutured Floer homology and the equivalence of Seifert surfaces
}

\author{
MATTHEW HEDDEN \\ ANDRÁS JUHÁSZ \\ SUCHARIT SARKAR
}

\begin{abstract}
The goal of this paper is twofold. First, given a Seifert surface $R$ in the 3-sphere, we show how to construct a Heegaard diagram for the sutured manifold $S^{3}(R)$ complementary to $R$, which in turn enables us to compute the sutured Floer homology of $S^{3}(R)$ combinatorially. Secondly, we outline how the sutured Floer homology of $S^{3}(R)$, together with the Seifert form of $R$, can be used to decide whether two minimal genus Seifert surfaces of a given knot are isotopic in $S^{3}$. We illustrate our techniques by showing that the knot $8_{3}$ has two minimal genus Seifert surfaces up to isotopy. Furthermore, for any $n \geq 1$ we exhibit a knot $K_{n}$ that has at least $n$ nonisotopic free minimal genus Seifert surfaces.
\end{abstract}

57M27; 57R58

\section{Introduction}

It is well-known that every knot in the three-sphere bounds an embedded orientable surface. The various surfaces which a given knot bounds are called Seifert surfaces and play an important role in knot theory and low-dimensional topology as a whole. Given a knot $K$, the minimum genus of any Seifert surface for $K$ is called the genus of $K$. The genus of a knot is a fundamental invariant, and minimal genus Seifert surfaces tell us a lot about the topological and geometric properties of a knot. In particular, the only knot of genus zero is the unknot.

Sutured manifolds were defined by Gabai [5] to study the genera of knots and links. Roughly speaking, a sutured manifold is a pair $(M, \gamma)$, where $M$ is a compact oriented 3 -manifold with boundary, $\gamma$ is a set of thickened oriented simple closed curves in $\partial M$ that splits $\partial M$ into two parts $R_{+}(\gamma)$ and $R_{-}(\gamma)$; finally, $\gamma$ is oriented as the boundary of $R_{+}(\gamma)$. Here the components of $\gamma$ are called sutures. Given a Seifert surface $R$ in $S^{3}$, one obtains a sutured manifold $S^{3}(R)=(M, \gamma)$ by taking $M=S^{3} \backslash \operatorname{Int}(R \times I)$ and $\gamma=\partial R \times I$. A sutured manifold is called balanced if $\chi\left(R_{+}(\gamma)\right)=\chi\left(R_{-}(\gamma)\right)$, the manifold $M$ is irreducible and every component of $\partial M$ contains at least one suture. 
Sutured Floer homology is an invariant of balanced sutured manifolds introduced by the second author in [11] that generalizes the Ozsváth-Szabó invariants of closed 3-manifolds and links; see Ozsváth and Szabó [24; 25; 27] and Rasmussen [28]. A key feature of the Ozsváth-Szabó invariants is their ability to detect the genus of a knot $K$. The proof of this fact utilized sutured manifolds, but only so much as they were instrumental in providing taut foliations which allowed contact geometric and symplectic techniques to be employed [24].

With the advent of sutured Floer homology, a precise relationship between Gabai's machinery and Heegaard Floer homology has now been established; see the second author's $[11 ; 12 ; 13]$. Moreover, the genus detection of knot Floer homology has an elegant reinterpretation in this theory which we briefly explain.

In [12] it was shown that

$$
\operatorname{SFH}\left(S^{3}(R)\right) \cong \widehat{H F K}(K, g(R)) .
$$

Here, the right hand side is the knot Floer homology group of $K$ supported in Alexander grading $g(R)$; see [25]. This isomorphism was then used, together with further properties of $S F H$ and results of Gabai, to reprove (among many other things) the fact that knot Floer homology detects the genus. A striking aspect of this new proof is that it completely bypasses the four-dimensional methods which were originally used.

Even though $\operatorname{SFH}\left(S^{3}(R)\right)$ is isomorphic to the top term of knot Floer homology $\widehat{H F K}(K, g(R))$, the former carries a grading along relative $\operatorname{Spin}^{c}$-structures on $M$. Note that $\operatorname{Spin}^{c}(M, \gamma)$ is an affine space over $H_{1}(M)$, so the reader is free to regard this as a relative $H_{1}(M)$ grading. Given a knot $K$, this grading does depend on the choice of Seifert surface for $K$; see Altman [2]. One of the goals of this paper is to determine $\operatorname{SFH}\left(S^{3}(R)\right)$ together with the $\operatorname{Spin}^{c}$-grading. The only missing step is to construct a Heegaard diagram for the sutured manifold $S^{3}(R)$. From there, the sutured Floer homology can be computed using a generalization of the Sarkar-Wang algorithm described in [12].

Note that for a $\operatorname{Spin}^{c}$-structure $\mathfrak{s} \in \operatorname{Spin}^{c}(M, \gamma)$ and for a fixed homology orientation of $H_{*}\left(M, R_{-}(\gamma)\right)$ the group $\operatorname{SFH}(M, \gamma, \mathfrak{s})$ is $\mathbb{Z}_{2}$-graded. It was shown in Friedl, Juhász and Rasmussen [4] that $\chi(\operatorname{SFH}(M, \gamma, \mathfrak{s}))=T_{(M, \gamma)}(\mathfrak{s})$, where

$$
T_{(M, \gamma)}: \operatorname{Spin}^{c}(M, \gamma) \longrightarrow \mathbb{Z}
$$

is a type of relative Turaev torsion that is easy to compute using Fox calculus. Furthermore, if $R$ is the Seifert surface of an alternating knot in $S^{3}$, then by [4, Corollary 6] the group $\operatorname{SFH}\left(S^{3}(R), \mathfrak{s}\right)$ is either zero or $\mathbb{Z}$, so it is completely determined by the 
torsion $T_{S^{3}(R)}(\mathfrak{s})$. This provides another method for computing $\operatorname{SFH}\left(S^{3}(R)\right)$ when the knot $\partial R$ is alternating.

It is natural to ask what information $\operatorname{SFH}\left(S^{3}(R)\right)$ carries about the Seifert surface $R$. Our second aim is to show that the $\operatorname{Spin}^{c}$-grading on $\operatorname{SFH}(M, \gamma)$, together with the Seifert form of $R$, can be used to distinguish two minimal genus Seifert surfaces of a given knot. Of course, we have to first specify what kind of equivalence we are using. There are several natural candidates and many examples are known of knots with inequivalent Seifert surfaces for each notion; see Alford [1], Eisner [3], Kakimizu [14; 15], Kobayashi [16] and Lyon [18]. One could say that the Seifert surfaces $R_{1}$ and $R_{2}$ of a knot $K$ are equivalent if

(1) $S^{3} \backslash R_{1}$ and $S^{3} \backslash R_{2}$ are homeomorphic,

(2) the sutured manifolds $S^{3}\left(R_{1}\right)$ and $S^{3}\left(R_{2}\right)$ are homeomorphic,

(3) there is an orientation preserving homeomorphism of $S^{3}$ taking $R_{1}$ to $R_{2}$ (weak equivalence),

(4) $R_{1}$ and $R_{2}$ are isotopic in the knot complement (strong equivalence).

Each of the above notions is stronger than the previous one. For us, weak and strong equivalence seem to be the most natural, we will discuss these further in Section 4. Note that the weak equivalence classes are the quotient of the strong equivalence classes by the action of the mapping class group of the knot complement. Together with normal surface theory, this provides an algorithm to classify minimal genus Seifert surfaces of a given knot up to weak equivalence: list all minimal genus surfaces in the knot complement (possibly with multiplicities), solve the isotopy problem and determine the action of the mapping class group of the knot complement on the strong equivalence classes.

The above algorithm is rather impractical to implement by hand and it is useful to have an invariant which can quickly determine if two minimal genus Seifert surfaces are weakly equivalent. To this end, the sutured Floer homology $\operatorname{SFH}\left(S^{3}(R)\right)$, and often the much simpler relative torsion invariant $T_{S^{3}(R)}$, can distinguish surfaces up to equivalence (2). There are Seifert surfaces, however, whose complementary sutured manifolds are homeomorphic but which are not weakly equivalent. Indeed, the surfaces of Theorem 1.1 below fall into this category. To detect this distinction, we must account for how $R_{+}(\gamma)$ and $R_{-}(\gamma)$ are glued together. The Seifert form of $R$ contains information about this gluing, and used in conjunction with the torsion $T_{S^{3}(R)}$, we can often effectively distinguish Seifert surfaces up to weak equivalence. Of course, the combination of $S F H$ with the Seifert form provides a more powerful technique, but is harder to implement. 
Let us now outline our method. The difference between any two $\operatorname{Spin}^{c}$-structures supporting nonzero sutured Floer homology yields an element of $H_{1}(M)$. This element can be explicitly identified from the Heegaard diagrams we produce. However, it is difficult to determine whether $\alpha_{1} \in H_{1}\left(M_{1}\right)$ and $\alpha_{2} \in H_{1}\left(M_{2}\right)$ presented by Heegaard diagrams for $M_{1}$ and $M_{2}$, respectively, are identical (in the presence of an assumed equivalence between $M_{1}$ and $\left.M_{2}\right)$. In the present context, the key observation is that $H_{1}\left(S^{3} \backslash \operatorname{Int}(R \times I)\right) \cong H_{1}(R)$. This isomorphism equips the former group with a bilinear form; namely, the Seifert form on $R$. We can use this form to distinguish elements of $H_{1}\left(S^{3} \backslash \operatorname{Int}\left(R_{i} \times I\right)\right)$ obtained as differences of $\operatorname{Spin}^{c}$-structures supporting nontrivial Floer homology. Distinguishing these elements, in turn, shows that the sutured manifolds are not equivalent and hence the Seifert surfaces are not isotopic.

To illustrate our algorithm of drawing Heegaard diagrams of Siefert surface complements, and also our method to distinguish two minimal genus Seifert surfaces of a knot up to weak equivalence, we show that the knot $8_{3}$ has precisely two minimal genus Seifert surfaces $R_{1}$ and $R_{2}$ up to weak equivalence. Of course, in such a simple example other techniques could be applied, but we find this example particularly enlightening due to the fact that the complementary sutured manifolds $S^{3}\left(R_{1}\right)$ and $S^{3}\left(R_{2}\right)$ are homeomorphic. Thus both $S F H$ and the Seifert form have to be used to distinguish $R_{1}$ and $R_{2}$. In this example, the torsion would have sufficed; see [4, Example 8.3]. However, we still compute $S F H$ to demonstrate how to draw the Heegaard diagrams. Employing the same techniques, we get the following.

Theorem 1.1 For any $n \geq 1$, there exists a knot $K_{n}$ with free Seifert surfaces $\left\{F_{0}, \ldots, F_{n}\right\}$, such that $F_{i}$ is not isotopic to $F_{j}$ for any $i \neq j$.

We conclude by pointing out that the first version of this paper appeared some time before [2] and [4], and part of the original motivation was the question (resolved in [2]) of whether the $\operatorname{Spin}^{c}$-grading on $\operatorname{SFH}\left(S^{3}(R)\right)$ depends on the minimal genus Seifert surface $R$.

Acknowledgment It is our pleasure to thank David Gabai, Eli Grigsby, Chuck Livingston and Zoltán Szabó for their interest in this work and many helpful conversations. The first author was supported by NSF grants number DMS0706979 and DMS0906258, NSF CAREER grant number DMS-1150872 and an Alfred P Sloan Research Fellowship. The second author was supported by a Royal Society Research Fellowship and OTKA grant number NK81203. The third author was partially funded by a Princeton Honorific Fellowship, a Princeton Centennial Fellowship and a Clay Mathematics Institute Postdoctoral fellowship. 


\section{Preliminaries}

Sutured manifolds were introduced by Gabai in [6]. They provide a natural framework for constructing taut foliations on three-manifolds via inductive cut-and-paste procedures. The motivation for taut foliations, in turn, is that they tell us about the Thurston norm [30] of three-manifolds. In particular, they can be used to determine the genera of knots. Sutured Floer homology is a generalization of Ozsváth-Szabó Floer homology to an invariant of sutured manifolds, and was defined the second author in [11]. Its definition and study were motivated by a desire to clarify and further explore connections between the Ozsváth-Szabó invariants and Gabai's theory hinted at by the results in [24]. In particular, a primary goal was to show that knot Floer homology detects fibered knots; see Ghiggini [10], Ni [21; 22] and the second author's [12; 13].

In this section, we begin by briefly recalling some basic notions from the theory of sutured manifolds. We then discuss sutured Floer homology, paying particular attention to sutured Heegaard diagrams. These diagrams are the input for the sutured Floer homology invariants. Special focus will be given to sutured Heegaard diagrams adapted to a decomposing surface and the way in which decomposition of sutured manifolds is understood in terms of these diagrams.

We refer the reader to Gabai $[6 ; 7 ; 9]$ for more details on sutured manifolds, and to $[11 ; 12]$ for details on sutured Floer homology.

\subsection{Sutured manifolds}

The cornerstone of Gabai's machinery is the notion of a sutured manifold.

Definition 2.1 A sutured manifold $(M, \gamma)$ is a compact oriented 3-manifold with boundary, $(M, \partial M)$, together with a set $\gamma \subset \partial M$ of pairwise disjoint annuli $A(\gamma)$ and tori $T(\gamma)$. Furthermore, the interior of each component of $A(\gamma)$ contains a suture, ie a homologically nontrivial oriented simple closed curve. We denote the union of the sutures by $s(\gamma)$.

Finally, every component of $R(\gamma)=\partial M \backslash \operatorname{Int}(\gamma)$ is required to be oriented. Define $R_{+}(\gamma)$ (respectively $R_{-}(\gamma)$ ) to be those components of $\partial M \backslash \operatorname{Int}(\gamma)$ whose normal vectors point out of (respectively into) $M$. The orientation on $R(\gamma)$ must be coherent with respect to $s(\gamma)$, ie if $\delta$ is a component of $\partial R(\gamma)$ and is given the boundary orientation, then $\delta$ must represent the same homology class in $H_{1}(\gamma)$ as some suture.

Definition 2.2 Two sutured manifolds $\left(M_{1}, \gamma_{1}\right),\left(M_{2}, \gamma_{2}\right)$ are said to be equivalent if there is an orientation-preserving diffeomorphism $f: M_{1} \rightarrow M_{2}$ which restricts to an orientation-preserving diffeomorphism between $R\left(\gamma_{1}\right)$ and $R\left(\gamma_{2}\right)$. 
Definition 2.3 A sutured manifold $(M, \gamma)$ is called balanced if $\mathrm{M}$ has no closed components, $\chi\left(R_{+}(\gamma)\right)=\chi\left(R_{-}(\gamma)\right)$ and the map $\pi_{0}(A(\gamma)) \rightarrow \pi_{0}(\partial M)$ is surjective.

The following two examples can be found in [8].

Example 2.4 Let $R$ be a compact oriented surface with no closed components. Then there is an induced orientation on $\partial R$. Let $M=R \times I$, define $\gamma=\partial R \times I$ and finally put $s(\gamma)=\partial R \times\{1 / 2\}$. The balanced sutured manifold $(M, \gamma)$ obtained by this construction is called a product sutured manifold.

Example 2.5 Let $Y$ be a closed connected oriented 3-manifold and let $R \subset Y$ be a compact oriented surface with no closed components. We define a sutured manifold $Y(R)=(M, \gamma)$ to be the sutured manifold where $M=Y \backslash \operatorname{Int}(R \times I)$, with the suture $\gamma=\partial R \times I$. Furthermore $s(\gamma)=\partial R \times\{1 / 2\}$.

From the perspective of Floer homology, the following example is also quite relevant.

Example 2.6 Let $K \subset Y$ be a knot, and let $Y_{2 n}(K)=\left(M, \gamma_{2 n}\right)$ denote the sutured manifold with $M=Y \backslash v(K)$ the knot exterior and $s\left(\gamma_{2 n}\right)$ consisting of $2 n$ parallel copies of the meridian of $K$, with orientations alternating.

The key to Gabai's inductive procedures is the concept of a sutured manifold decomposition, which we now recall. See [6, Definition 3.1] and [9, Correction 0.3]. We begin with the notion of a decomposing surface.

Definition 2.7 Let $(M, \gamma)$ be a sutured manifold. A decomposing surface is an oriented, properly embedded surface $S \subset M$, such that no component of $\partial S$ bounds a disk in $R(\gamma)$ and no component of $S$ is a disk $D$ with $\partial D \subset R(\gamma)$. Moreover, for every component $\lambda$ of $S \cap \gamma$ one of (1)-(3) holds:

(1) $\lambda$ is a properly embedded nonseparating arc in $\gamma$ satisfying $|\lambda \cap s(\gamma)|=1$.

(2) $\lambda$ is a simple closed curve in an annular component $A$ of $\gamma$ in the same homology class as $A \cap s(\gamma)$.

(3) $\lambda$ is a homotopically nontrivial curve in a torus component $T$ of $\gamma$, and if $\delta$ is another component of $T \cap S$, then $\lambda$ and $\delta$ represent the same homology class in $H_{1}(T)$. 
A decomposing surface $S$ defines a sutured manifold decomposition, denoted

$$
(M, \gamma) \stackrel{S}{\sim}\left(M^{\prime}, \gamma^{\prime}\right),
$$

where

$$
\begin{aligned}
M^{\prime} & =M \backslash \operatorname{Int}(N(S)), \\
\gamma^{\prime} & =\left(\gamma \cap M^{\prime}\right) \cup N\left(S_{+}^{\prime} \cap R_{-}(\gamma)\right) \cup N\left(S_{-}^{\prime} \cap R_{+}(\gamma)\right), \\
R_{+}\left(\gamma^{\prime}\right) & =\left(\left(R_{+}(\gamma) \cap M^{\prime}\right) \cup S_{+}^{\prime}\right) \backslash \operatorname{Int}\left(\gamma^{\prime}\right), \\
R_{-}\left(\gamma^{\prime}\right) & =\left(\left(R_{-}(\gamma) \cap M^{\prime}\right) \cup S_{-}^{\prime}\right) \backslash \operatorname{Int}\left(\gamma^{\prime}\right) .
\end{aligned}
$$

Here $S_{+}^{\prime}$ (respectively $S_{-}^{\prime}$ ) is the component of $\partial N(S) \cap M^{\prime}$ whose normal vector points out of (respectively into) $M^{\prime}$.

Remark 2.8 In other words, the sutured manifold $\left(M^{\prime}, \gamma^{\prime}\right)$ is constructed by splitting $M$ along $S$, creating $R_{+}\left(\gamma^{\prime}\right)$ by adding $S_{+}^{\prime}$ to what is left of $R_{+}(\gamma)$ and creating $R_{-}\left(\gamma^{\prime}\right)$ by adding $S_{-}^{\prime}$ to what is left of $R_{-}(\gamma)$. Finally, one creates the annuli of $\gamma^{\prime}$ by "thickening" $R_{+}\left(\gamma^{\prime}\right) \cap R_{-}\left(\gamma^{\prime}\right)$.

The following lemma indicates that Examples 2.5 and 2.6 are connected by a sutured manifold decomposition.

Lemma 2.9 Suppose that $R$ is a Seifert surface for a knot $K \subset Y$. Then

$$
Y_{2 n}(K) \stackrel{R}{\sim} Y(R) .
$$

\subsection{Sutured Floer homology}

We can associate to a balanced sutured manifold a collection of abelian groups, called the sutured Floer homology groups [11]. These groups are the homology groups of a chain complex, which is defined by a sutured Heegaard diagram. Sutured Heegaard diagrams generalize Heegaard diagrams of closed 3-manifolds so that we can also describe sutured manifolds.

Definition 2.10 A sutured Heegaard diagram is a tuple $(\Sigma, \boldsymbol{\alpha}, \boldsymbol{\beta})$, where $\Sigma$ is a compact oriented surface with boundary and $\boldsymbol{\alpha}=\left\{\alpha_{1}, \ldots, \alpha_{m}\right\}$ and $\boldsymbol{\beta}=\left\{\beta_{1}, \ldots, \beta_{n}\right\}$ are two sets of pairwise disjoint simple closed curves in $\operatorname{Int}(\Sigma)$.

Every sutured Heegaard diagram $(\Sigma, \boldsymbol{\alpha}, \boldsymbol{\beta})$ uniquely defines a sutured manifold $(M, \gamma)$ using the following construction: Let $M$ be the 3 -manifold obtained from $\Sigma \times I$ 
by attaching 3-dimensional 2-handles along the curves $\alpha_{i} \times\{0\}$ and $\beta_{j} \times\{1\}$ for $i=1, \ldots, m$ and $j=1, \ldots, n$. The sutures are defined by taking $\gamma=\partial M \times I$ and $s(\gamma)=\partial M \times\{1 / 2\}$.

Definition 2.11 A sutured Heegaard diagram $(\Sigma, \boldsymbol{\alpha}, \boldsymbol{\beta})$ is called balanced if $|\boldsymbol{\alpha}|=|\boldsymbol{\beta}|$ and the maps $\pi_{0}(\partial \Sigma) \rightarrow \pi_{0}(\Sigma \backslash \bigcup \alpha)$ and $\pi_{0}(\partial \Sigma) \rightarrow \pi_{0}(\Sigma \backslash \bigcup \beta)$ are surjective.

The following is [11, Proposition 2.14].

Proposition 2.12 For every balanced sutured manifold $(M, \gamma)$ there exists a balanced diagram defining it.

In order to understand how $S F H$ behaves under surface decompositions, it is necessary to understand these operations at the level of Heegaard diagrams. To this end, we have the following definition [12, Definition 4.3].

Definition 2.13 A balanced diagram adapted to the decomposing surface $R$ in $(M, \gamma)$ is a quadruple

$$
(\Sigma, \boldsymbol{\alpha}, \boldsymbol{\beta}, P),
$$

satisfying the following conditions:

(1) $(\Sigma, \boldsymbol{\alpha}, \boldsymbol{\beta})$ is a balanced diagram of $(M, \gamma)$.

(2) $P \subset \Sigma$ is a quasipolygon (ie a closed subsurface of $\Sigma$ whose boundary is a union of polygons) such that $P \cap \partial \Sigma$ is exactly the set of vertices of $P$.

(3) There is a decomposition $\partial P=A \cup B$, where both $A$ and $B$ are unions of pairwise disjoint edges of $P$ satisfying $\alpha \cap B=\varnothing$ and $\beta \cap A=\varnothing$ for every $\alpha \in \boldsymbol{\alpha}$ and $\beta \in \boldsymbol{\beta}$.

(4) $R$ is obtained, up to equivalence, by smoothing the corners of the surface $(P \times\{1 / 2\}) \cup(A \times[1 / 2,1]) \cup(B \times[0,1 / 2]) \subset(M, \gamma)$ (recall the construction following Definition 2.10).

(5) The orientation of $R$ is given by the orientation of $P \subset \Sigma$.

We will frequently refer to a diagram adapted to $R$ as a surface diagram. A surface diagram allows us to represent decomposition along $R$ in terms of Heegaard diagrams. To describe this process, let $(\Sigma, \boldsymbol{\alpha}, \boldsymbol{\beta}, P)$ be a surface diagram for $R$. To such a diagram, we can uniquely associate a six-tuple

$$
D(P)=\left(\Sigma^{\prime}, \boldsymbol{\alpha}^{\prime}, \boldsymbol{\beta}^{\prime}, P_{A}, P_{B}, p\right) .
$$


Here, $\left(\Sigma^{\prime}, \boldsymbol{\alpha}^{\prime}, \boldsymbol{\beta}^{\prime}\right)$ is a balanced diagram, $p: \Sigma^{\prime} \rightarrow \Sigma$ is a smooth map, and $P_{A}, P_{B} \subset \Sigma^{\prime}$ are two closed subsurfaces (see Figure 2.1). We will refer to $D(P)$ as the decomposed diagram. $D(P)$ is constructed as follows.

We begin with $\Sigma^{\prime}$. Let $P_{A}$ and $P_{B}$ be two disjoint copies of $P$, together with diffeomorphisms $p_{A}: P_{A} \rightarrow P$ and $p_{B}: P_{B} \rightarrow P$. Then

$$
\Sigma^{\prime}=P_{A} \bigsqcup_{p_{A}^{-1}(A) \leftrightarrow A} \overline{(\Sigma \backslash P)} \bigsqcup_{p_{B}^{-1}(B) \leftrightarrow B} P_{B} .
$$

Thus, $\Sigma^{\prime}$ is obtained by removing $P$ from $\Sigma$ and then gluing two copies of $P$ to the closure of the remaining surface, one copy glued along its $A$ edges and the other along its $B$ edges.

The map $p: \Sigma^{\prime} \rightarrow \Sigma$ agrees with $p_{A}$ on $P_{A}$ and $p_{B}$ on $P_{B}$, and it maps $\Sigma^{\prime} \backslash\left(P_{A} \cup P_{B}\right)$ to $\Sigma \backslash P$ using the obvious diffeomorphism.

Finally, let

$$
\boldsymbol{\alpha}^{\prime}=\left\{p^{-1}(\alpha) \backslash P_{B}: \alpha \in \boldsymbol{\alpha}\right\} \quad \text { and } \quad \boldsymbol{\beta}^{\prime}=\left\{p^{-1}(\beta) \backslash P_{A}: \beta \in \boldsymbol{\beta}\right\} .
$$

Thus $p$ is $1: 1$ over $\Sigma \backslash P$, is $2: 1$ over $P$ and $\alpha$ curves are lifted to $P_{A}$ and $\beta$ curves to $P_{B}$. For the purposes of sutured Floer homology computations it is useful to note that, given a conformal structure on $\Sigma$, there is a unique conformal structure on $\Sigma^{\prime}$ making $p$ into a conformal map. The following proposition indicates that the decomposed diagram produces a Heegaard diagram for the sutured manifold obtained by decomposing along $R$.

Proposition 2.14 [12, Proposition 5.2] Let $(M, \gamma)$ be a balanced sutured manifold and

$$
(M, \gamma) \stackrel{S}{\sim}\left(M^{\prime}, \gamma^{\prime}\right)
$$

a surface decomposition. If $(\Sigma, \boldsymbol{\alpha}, \boldsymbol{\beta}, P)$ is a surface diagram adapted to $S$ and if

$$
D(P)=\left(\Sigma^{\prime}, \boldsymbol{\alpha}^{\prime}, \boldsymbol{\beta}^{\prime}, P_{A}, P_{B}, p\right)
$$

is the decomposed diagram, then $\left(\Sigma^{\prime}, \boldsymbol{\alpha}^{\prime}, \boldsymbol{\beta}^{\prime}\right)$ is a balanced diagram defining $\left(M^{\prime}, \gamma^{\prime}\right)$.

2.2.1 The sutured Floer chain complex We conclude this section by briefly recalling the definition of the sutured Floer chain complex and describing the splitting of this complex along relative $\operatorname{Spin}^{c}$-structures.

Given a balanced sutured Heegaard diagram $(\Sigma, \boldsymbol{\alpha}, \boldsymbol{\beta})$ for a balanced sutured manifold $(M, \gamma)$, one can define a chain complex $(C(\Sigma, \boldsymbol{\alpha}, \boldsymbol{\beta}), \partial)$. As a $\mathbb{Z} / 2 \mathbb{Z}$-vector space, 


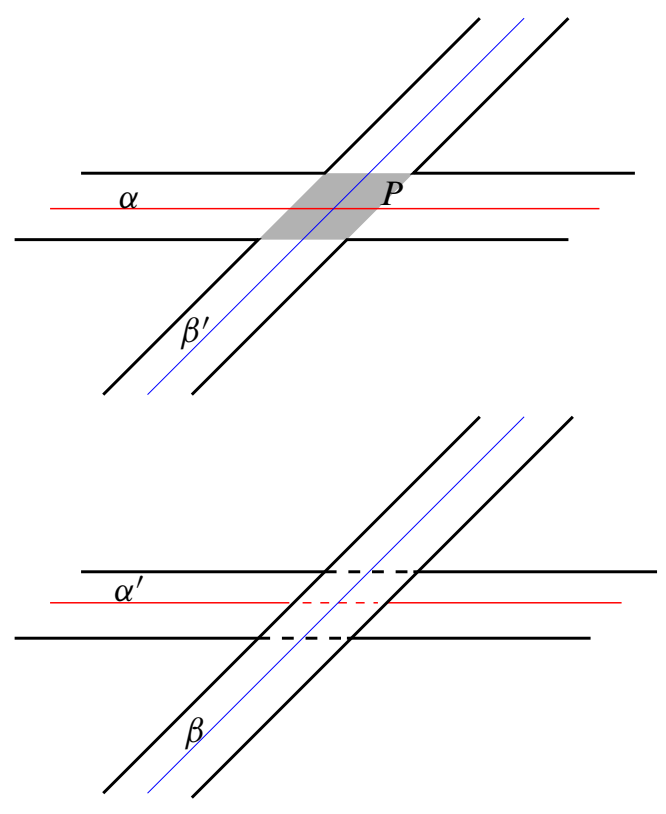

Figure 2.1: Balanced diagram before and after a surface decomposition

$C(\Sigma, \boldsymbol{\alpha}, \boldsymbol{\beta})$ is generated by $k$-tuples $\boldsymbol{x}=x_{1} \times \cdots \times x_{k}$ of intersection points, where $x_{i} \in \alpha_{i} \cap \beta_{\sigma(i)}$ (here $\sigma$ is a permutation in the symmetric group on $k$ letters and $k=|\boldsymbol{\alpha}|=|\boldsymbol{\beta}|$ is the number of $\alpha$ curves). If $k=0$, then despite having no curves we have a single generator (for the familiar reader, this is due to the fact that the 0 -th symmetric product of $\Sigma$ is a point, which coincides with the intersection of the two Lagrangians).

The chain complex is equipped with a differential $\partial$ that counts points in moduli spaces of certain pseudoholomorphic maps; see Lipshitz [17] and Ozsváth-Szabó [27]. To describe this, let us call the closures of the connected components of $\Sigma-\boldsymbol{\alpha}-\boldsymbol{\beta}$ regions, and denote them by $\mathcal{D}_{1}, \ldots, \mathcal{D}_{j}$. Given two generators $\boldsymbol{x}, \boldsymbol{y} \in C(\Sigma, \boldsymbol{\alpha}, \boldsymbol{\beta})$, consider a linear combination of regions

$$
\phi=\sum_{i=1}^{j} n_{i} \cdot \mathcal{D}_{i}
$$

which satisfies $\partial\left(\left.\partial \phi\right|_{\alpha}\right)=\boldsymbol{y}-\boldsymbol{x}$, ie the oriented boundary of the $\alpha$ components of $\partial \phi$ consists of the $k$-tuples of intersection points which comprise $-\boldsymbol{x}$ and $\boldsymbol{y}$. If, furthermore, $\phi \cap \partial \Sigma=\varnothing$, we say that $\phi$ is a domain connecting $\boldsymbol{x}$ to $\boldsymbol{y}$. Let us denote by $\pi_{2}(\boldsymbol{x}, \boldsymbol{y})$ the set of domains connecting $\boldsymbol{x}$ to $\boldsymbol{y}$. 
We define an endomorphism $\partial$ of $C(\Sigma, \boldsymbol{\alpha}, \boldsymbol{\beta})$ by specifying it on generators:

$$
\partial \boldsymbol{x}=\sum_{\boldsymbol{y} \in C(\Sigma, \boldsymbol{\alpha}, \boldsymbol{\beta})} \sum_{\left\{\phi \in \pi_{2}(\boldsymbol{x}, \boldsymbol{y}) \mid \mu(\phi)=1\right\}} \# \widehat{\mathcal{M}}(\phi) \cdot \boldsymbol{y} .
$$

In the formula, $\# \widehat{\mathcal{M}}(\phi)$ denotes the number (modulo 2 ) of unparameterized pseudoholomorphic maps of the unit disk $D^{2} \subset \mathbb{C}$ into the $k$-fold symmetric product of $\Sigma$, satisfying boundary conditions specified by $(\boldsymbol{\alpha}, \boldsymbol{\beta}, \boldsymbol{x}, \boldsymbol{y})$ and whose homotopy class is determined by $\phi$. The quantity $\mu(\phi)$ is the Maslov index of the domain, $\phi$, and the condition $\mu(\phi)=1$ is in place to ensure that the count can be performed (ie there exist only finitely many). We refer the reader to [11] for more details on the definition of $\partial$, but do call to mind the following important property (see [27, Lemma 3.2]).

Lemma 2.15 Let $\phi=\sum_{i=1}^{j} n_{i} \cdot \mathcal{D}_{i}$ be a domain. If $\# \widehat{\mathcal{M}}(\phi) \neq 0$, then $n_{i} \geq 0$ for all $i$.

For the purposes of computation, it is also useful to know that $\partial$ can be reformulated in terms of counting holomorphic maps of surfaces with boundary (and with marked points on the boundary) into $\Sigma \times D^{2}$. This is made precise in [17].

The following is contained in [11, Theorems 7.1 and 7.5].

Theorem 2.16 Let $(\Sigma, \boldsymbol{\alpha}, \boldsymbol{\beta})$ be a Heegaard diagram for the sutured manifold $(M, \gamma)$, and let $(C(\Sigma, \boldsymbol{\alpha}, \boldsymbol{\beta}), \partial)$ be as above. Then $\partial^{2}=0$. The resulting homology group is denoted $\operatorname{SFH}(M, \gamma)$. If the sutured manifolds $\left(M_{1}, \gamma_{1}\right)$ and $\left(M_{2}, \gamma_{2}\right)$ are equivalent, then $\operatorname{SFH}\left(M_{1}, \gamma_{1}\right)$ and $\operatorname{SFH}\left(M_{2}, \gamma_{2}\right)$ are isomorphic.

The above theorem suppresses some extra structure which we now discuss; namely, the splitting of sutured Floer homology into subgroups indexed by the set of relative Spin ${ }^{c}$-structures on $(M, \gamma)$, which we denote $\operatorname{Spin}^{c}(M, \gamma)$. Indeed, to a generator $\boldsymbol{x} \in C(\Sigma, \boldsymbol{\alpha}, \boldsymbol{\beta})$ one can associate a relative $\operatorname{Spin}^{c}$-structure, $\mathfrak{s}(\boldsymbol{x}) \in \operatorname{Spin}^{c}(M, \gamma)$, as follows.

First, pick a Morse function which determines the Heegaard diagram and whose gradient vector field points into $M$ along $R_{-}(\gamma)$, points out of $M$ along $R_{+}(\gamma)$, and which is the gradient of the height function $s(\gamma) \times I \rightarrow I$ on $\gamma$. Next, modify the gradient field in a neighborhood of flowlines specified by $x_{i} \in \boldsymbol{x}$. This produces a nonvanishing vector field $v$ with prescribed behavior on $\partial M$. The homology class of $v$ (in the sense of Turaev [32]) specifies a relative $\operatorname{Spin}^{c}-$ structure, which we denote by $\mathfrak{s}(\boldsymbol{x})$. The "relative" terminology arises since we require vector fields to have prescribed behavior on $\partial M$. See $[11$, Section 4] for more details. 
For our purposes, the most important aspect of $\operatorname{Spin}^{c}(M, \gamma)$ is that it is an affine set for $H^{2}(M, \partial M ; \mathbb{Z})$. This implies, in particular, that we can talk about the difference of two relative $\operatorname{Spin}^{c}$-structures, $\mathfrak{s}(\boldsymbol{x})-\mathfrak{s}(\boldsymbol{y}) \in H^{2}(M, \partial M ; \mathbb{Z})$. Given two generators $\boldsymbol{x}$ and $\boldsymbol{y}$, we can concretely determine $\mathfrak{s}(\boldsymbol{x})-\mathfrak{s}(\boldsymbol{y})$ as follows. First pick a collection of $k$ oriented subarcs of the $\alpha$ curves, $\gamma_{\alpha} \subset \boldsymbol{\alpha}$, which connect the intersection points $x_{i}$ to $y_{i}$. Similarly, pick a collection of $k$ oriented subarcs of the $\beta$ curves, $\gamma_{\beta} \subset \boldsymbol{\beta}$, which connect the intersection points $y_{i}$ to $x_{\sigma(i)}$ for some permutation $\sigma$. The sum $\gamma_{\boldsymbol{x}, \boldsymbol{y}}=\gamma_{\alpha}+\gamma_{\beta}$ is a collection of oriented closed curves in $\Sigma \subset M$ whose homology class we denote by $\epsilon(\boldsymbol{x}, \boldsymbol{y}) \in H_{1}(M ; \mathbb{Z})$. The following lemma will be useful.

Lemma 2.17 [11, Lemma 4.7] Let $\boldsymbol{x}, \boldsymbol{y} \in C(\Sigma, \boldsymbol{\alpha}, \boldsymbol{\beta})$ be generators. Then

$$
\mathfrak{s}(\boldsymbol{x})-\mathfrak{s}(\boldsymbol{y})=\operatorname{PD}[\epsilon(\boldsymbol{x}, \boldsymbol{y})] \in H^{2}(M, \partial M ; \mathbb{Z}),
$$

where $\operatorname{PD}[\epsilon(\boldsymbol{x}, \boldsymbol{y})]$ denotes the Poincaré dual of $\epsilon(\boldsymbol{x}, \boldsymbol{y})$.

The lemma makes clear the claim from the introduction; namely, that $(C(\Sigma, \boldsymbol{\alpha}, \boldsymbol{\beta}), \partial)$ splits as a direct sum of complexes which are indexed by relative $\operatorname{Spin}^{c}$-structures. To see this, first observe that $(C(\Sigma, \boldsymbol{\alpha}, \boldsymbol{\beta}), \partial)$ splits into subcomplexes corresponding to the equivalence classes of the relation

$$
\boldsymbol{x} \sim \boldsymbol{y} \Longleftrightarrow \pi_{2}(\boldsymbol{x}, \boldsymbol{y}) \neq \varnothing .
$$

Next, note that if $\phi \in \pi_{2}(\boldsymbol{x}, \boldsymbol{y})$, then $\epsilon(\boldsymbol{x}, \boldsymbol{y})=[\partial \phi]=0 \in H_{1}(M ; \mathbb{Z})$. Thus, if $\boldsymbol{x}$ and $\boldsymbol{y}$ are in the same subcomplex, they represent the same $\operatorname{Spin}^{c}$-structure. Conversely, if $\boldsymbol{x}$ and $\boldsymbol{y}$ represent the same $\operatorname{Spin}^{c}$-structure, then $\epsilon(\boldsymbol{x}, \boldsymbol{y})=0 \in H_{1}(M ; \mathbb{Z})$. In light of the isomorphism

$$
H_{1}(M ; \mathbb{Z}) \cong \frac{H_{1}(\Sigma ; \mathbb{Z})}{\operatorname{Span}\left(\left[\alpha_{1}\right], \ldots,\left[\alpha_{d}\right],\left[\beta_{1}\right], \ldots,\left[\beta_{d}\right]\right)},
$$

this implies that after possibly adding some copies of the $\alpha$ and $\beta$ curves to $\gamma_{\boldsymbol{x}}, \boldsymbol{y}$, we obtain a collection of curves which is null-homologous in $\Sigma$. A null-homology is an element $\phi \in \pi_{2}(\boldsymbol{x}, \boldsymbol{y})$.

We have the following refinement of the theorem stated above:

Theorem 2.18 Let $(M, \gamma)$ be a sutured manifold. Then

$$
\operatorname{SFH}(M, \gamma)=\bigoplus_{\mathfrak{s} \in \operatorname{Spin}^{c}(M, \gamma)} \operatorname{SFH}(M, \gamma, \mathfrak{s})
$$

The homology group $\operatorname{SFH}(M, \gamma, \mathfrak{s})$ depends only on the equivalence class of the sutured manifold and the relative $\mathrm{Spin}^{c}$-structure $\mathfrak{s}$, up to isomorphism. 
One of the most important aspects of sutured Floer homology is its behavior under surface decompositions, which we now describe. We will need a definition. As above, suppose we have a decomposition

$$
(M, \gamma) \stackrel{S}{\sim}\left(M^{\prime}, \gamma^{\prime}\right) .
$$

Let $(\Sigma, \boldsymbol{\alpha}, \boldsymbol{\beta}, P)$ be a surface diagram for $S$. Denote by $\mathcal{O}_{P} \subset \mathbb{T}_{\alpha} \cap \mathbb{T}_{\beta}$ the subset of generators, none of whose intersection points $x_{i} \in \boldsymbol{x}$ are contained in the quasipolygon $P \subset \Sigma$. Call such generators outer generators. The outer complex $C\left(\mathcal{O}_{P}\right) \subset C(\Sigma, \boldsymbol{\alpha}, \boldsymbol{\beta})$ is the subcomplex generated by $\mathcal{O}_{P}$. Finally, let $C\left(\Sigma^{\prime}, \boldsymbol{\alpha}^{\prime}, \boldsymbol{\beta}^{\prime}\right)$ be the chain complex associated to the decomposed diagram. The main result of [12] is the following.

Theorem 2.19 The outer complex $C\left(\mathcal{O}_{P}\right)$ forms a direct summand of $C(\Sigma, \boldsymbol{\alpha}, \boldsymbol{\beta})$. Moreover, the homology of $C\left(\mathcal{O}_{P}\right)$ is isomorphic to the homology of $C\left(\Sigma^{\prime}, \boldsymbol{\alpha}^{\prime}, \boldsymbol{\beta}^{\prime}\right)$. In particular,

$$
\operatorname{SFH}\left(M^{\prime}, \gamma^{\prime}\right):=H_{*}\left(C\left(\Sigma^{\prime}, \boldsymbol{\alpha}^{\prime}, \boldsymbol{\beta}^{\prime}\right)\right) \cong H_{*}\left(C\left(\mathcal{O}_{P}\right)\right) \leq \operatorname{SFH}(M, \gamma),
$$

where $\leq$ means "direct summand".

Sketch of proof Let $\mathcal{I}_{P}=\left(\mathbb{T}_{\alpha} \cap \mathbb{T}_{\beta}\right) \backslash \mathcal{O}_{P}$ be the set of inner generators. To see that $C\left(\mathcal{O}_{P}\right)$ forms a direct summand of $C F(\Sigma, \boldsymbol{\alpha}, \boldsymbol{\beta})$, we proceed by showing that $C\left(\mathcal{I}_{P}\right)$ is a complement of $C\left(\mathcal{O}_{P}\right)$. Consider generators $\boldsymbol{x} \in \mathcal{O}_{P}$ and $\boldsymbol{y} \in \mathcal{I}_{P}$. Then we will prove that both $\pi_{2}(\boldsymbol{x}, \boldsymbol{y})$ and $\pi_{2}(\boldsymbol{y}, \boldsymbol{x})$ are empty. The former implies that $\boldsymbol{y} \notin \partial \boldsymbol{x}$ for every $\boldsymbol{x} \in \mathcal{O}_{P}$, and repeating with each $\boldsymbol{y} \in \mathcal{I}_{P}$ we see that $\partial C\left(\mathcal{O}_{P}\right) \subset C\left(\mathcal{O}_{P}\right)$, ie $C\left(\mathcal{O}_{P}\right)$ is a subcomplex. Similarly, $\pi_{2}(\boldsymbol{y}, \boldsymbol{x})=\varnothing$ for every $\boldsymbol{x} \in \mathcal{O}_{P}$ and $\boldsymbol{y} \in \mathcal{I}_{P}$ implies that $C\left(\mathcal{I}_{P}\right)$ is also a subcomplex, hence $C\left(\mathcal{O}_{P}\right)$ is a quotient complex.

To see that $\pi_{2}(\boldsymbol{x}, \boldsymbol{y})=\varnothing$ for $\boldsymbol{x}, \boldsymbol{y}$ as above, consider the collection of curves $\gamma_{\boldsymbol{x}, \boldsymbol{y}}=$ $\gamma_{\alpha}+\gamma_{\beta}$ connecting $\boldsymbol{x}$ to $\boldsymbol{y}$. Pushing $\gamma_{\alpha}$ into the $\alpha$ handlebody and $\gamma_{\beta}$ into the $\beta$ handlebody, we obtain an oriented collection of curves $\tilde{\gamma}_{\boldsymbol{x}, \boldsymbol{y}} \subset M$. Note that since $\gamma_{\alpha}$ is oriented from $x_{i}$ to $y_{i}$, each intersection of $\tilde{\gamma}_{\boldsymbol{x}}, \boldsymbol{y}$ with the quasipolygon $P$ is positive. Since the only intersections $\tilde{\gamma}_{\boldsymbol{x}}, \boldsymbol{y} \cap S$ occur in $P$, this shows that $\#_{a l g}\left(\tilde{\gamma}_{\boldsymbol{x}}, \boldsymbol{y} \cap S\right)>0$. In particular $\epsilon(\boldsymbol{x}, \boldsymbol{y})=\left[\tilde{\gamma}_{\boldsymbol{x}}, \boldsymbol{y}\right] \neq 0$, showing that $\pi_{2}(\boldsymbol{x}, \boldsymbol{y})=\varnothing$. A similar argument implies that $\pi_{2}(\boldsymbol{y}, \boldsymbol{x})=\varnothing$.

It is immediate from the construction of the decomposed diagram that generators of $C\left(\Sigma^{\prime}, \boldsymbol{\alpha}^{\prime}, \boldsymbol{\beta}^{\prime}\right)$ are in bijection with $\mathcal{O}_{P}$. Indeed, since $\alpha$ and $\beta$ arcs in $P$ lift to $P_{A}$ and $P_{B}$ in the decomposed diagram, respectively, no intersection point $x_{i} \in P \subset \Sigma$ will lift to an intersection point in $\Sigma^{\prime}$ (since $P_{A} \cap P_{B}=\varnothing$ ). Hence any generator $\boldsymbol{x}$ containing $x_{i} \in P$ will not lift to a generator for $C\left(\Sigma^{\prime}, \boldsymbol{\alpha}^{\prime}, \boldsymbol{\beta}^{\prime}\right)$. On the other hand, 
the decomposed diagram is identical to the surface diagram (before decomposition) outside of $P$. Thus any outer generator lifts to a generator in $C\left(\Sigma^{\prime}, \boldsymbol{\alpha}^{\prime}, \boldsymbol{\beta}^{\prime}\right)$.

The most challenging part of the proof arises when showing that the differential on $C\left(\Sigma^{\prime}, \boldsymbol{\alpha}^{\prime}, \boldsymbol{\beta}^{\prime}\right)$ is identical to the differential on $C\left(\mathcal{O}_{P}\right)$ which it inherits as a subcomplex of $C(\Sigma, \boldsymbol{\alpha}, \boldsymbol{\beta})$. To prove this, [12] adapts the algorithm of [29] for computing Heegaard Floer homology to the context of sutured Floer homology. By making the surface diagram "nice", the count of pseudoholomorphic curves for each domain $\phi \in \pi_{2}(\boldsymbol{x}, \boldsymbol{y})$ with $\mu(\phi)=1$ can be done explicitly using the Riemann mapping theorem, together with the fact that pseudoholomorphic submanifolds of symplectic manifolds intersect positively. Moreover, for a nice enough surface diagram, the decomposed diagram will also be nice and one can explicitly identify the differentials for the respective complexes. See [12] for more details.

As a corollary, one obtains the theorem mentioned in the introduction, namely [12, Theorem 1.5].

Theorem 2.20 Let $R$ be a Seifert surface for a knot $K \subset S^{3}$. Then

$$
\operatorname{SFH}\left(S^{3}(R)\right) \cong \widehat{H F K}(K, g(R)),
$$

where the right hand side is the knot Floer homology group of $K$ supported in Alexander grading $g(R)[25]$.

Sketch of proof By Lemma 2.9, if we decompose $S_{2}^{3}(K)$ along $R$, then we get $S^{3}(R)$. Let $(\Sigma, \boldsymbol{\alpha}, \boldsymbol{\beta}, P)$ be a surface diagram adapted to $R$. The Alexander grading of a generator $\boldsymbol{x} \in C(\Sigma, \boldsymbol{\alpha}, \boldsymbol{\beta}) \cong \widehat{C F K}(K)$ can be defined as

$$
\frac{1}{2}\left\langle c_{1}(\mathfrak{s}(\boldsymbol{x})),[R, \partial R]\right\rangle,
$$

where $c_{1}(\mathfrak{s}(\boldsymbol{x})) \in H^{2}\left(S^{3} \backslash N(K), \partial ; \mathbb{Z}\right)$ is the relative Chern class of a relative $\operatorname{Spin}^{c}-$ structure associated to $\boldsymbol{x}$ (with respect to a particular trivialization of the restriction of $\mathfrak{s}(\boldsymbol{x})$ to the boundary) and $[R, \partial R] \in H_{2}\left(S^{3} \backslash N(K), \partial ; \mathbb{Z}\right)$ is the homology class of the surface. This evaluation, in turn, can be computed as

$$
\frac{1}{2}\left(\chi(R)-1+2 \#\left\{x_{i} \in \boldsymbol{x}: x_{i} \in P\right\}\right),
$$

where $P$ is the quasipolygon representing $R$ (see the proof of [25, Theorem 5.1] for motivation of this formula and [12] for precise details). Together with Theorem 2.19, this shows that

$$
\operatorname{SFH}\left(S^{3}(R)\right) \cong \widehat{H F K}(K,-g(R)) .
$$

However, $\widehat{H F K}(K, g(R)) \cong \widehat{H F K}(K,-g(R))$ by [25, Proposition 3.10]. 


\section{Constructing Heegaard diagrams adapted to a Seifert sur- face}

Given a Seifert surface $R$ for a knot $K \subset Y$, we wish to compute the sutured Floer homology groups $S F H(Y(R))$. Since these groups are the homology of a chain complex associated to a balanced sutured Heegaard diagram for $Y(R)$, it is necessary to produce such a diagram. To do this, recall that $Y(R)$ is obtained from the knot complement $Y_{2 n}(K)$ by decomposition along $R$. According to Section 2, it suffices to produce a balanced diagram for $Y_{2 n}(K)$ which is adapted to $R$. From there it is simple to obtain the decomposed diagram. This section will be dedicated to producing surface diagrams and clarifying the decomposed diagram which, by Proposition 2.14 , will necessarily be adapted to $Y(R)$. Throughout, we assume the genus of $R$ to be $g$.

A surface diagram for $R$ is, by definition, a Heegaard diagram for the sutured manifold associated to the knot complement which contains $R$ as a quasipolygon. Building this diagram requires two main steps:

(1) Construct a Heegaard diagram $(\Sigma, \boldsymbol{\alpha}, \boldsymbol{\beta})$ for the closed three-manifold $Y$ which contains $R$ as proper subsurface $R \subset \Sigma$.

(2) Remove disks from $\Sigma$ along $\partial R$, and modify the diagram by a sequence of isotopies and/or stabilizations to ensure that the diagram specifies $Y_{2 n}(K)$ and is adapted to $R$.

For any given Seifert surface, there are many ways to perform each step so we remain intentionally vague for the moment. The next two subsections discuss each step in detail. Indeed, for both steps we treat the case of a Seifert surface presented in an arbitrary manner. This has the advantage of being completely general and should thus be useful in a variety of situations.

For the sake of clarity, the third subsection presents explicit diagrams for the case of knots in $S^{3}$ with Seifert surfaces presented in a particularly appealing form. The presentation is analogous to a knot projection, and the surface diagrams which we produce can be viewed as the analogue of the diagrams used by Ozsváth and Szabó in [23] which connect the knot Floer homology chain complexes to Kauffman states.

\subsection{Constructing a diagram for $Y$ containing $R$}

Let $R \subset Y$ be a genus $g$ Seifert surface. We now describe the construction of a Heegaard diagram $(\Sigma, \boldsymbol{\alpha}, \boldsymbol{\beta})$ which contains $R$ as an embedded, proper subsurface of $\Sigma$. Similar diagrams have been useful in other contexts; see Ozsváth and Szabó [25; 26] and $\mathrm{Ni}[20]$. 
Begin with $R$. Now thicken to obtain $R \times I$. This is a handlebody of genus $2 g$. We can represent a basis for $H_{1}(R, \partial R ; \mathbb{Z})$ by $2 g$ pairwise disjoint properly embedded arcs $\gamma_{i}$ for $i=1, \ldots, 2 g$. Observe that $\gamma_{i} \times I$ is a properly embedded disk in the handlebody $R \times I$. Let $\beta_{i}=\partial\left(\gamma_{i} \times I\right)$. Note that $\partial(R \times I)$ consists of two copies of $R$, glued along $\partial R$. Thus $\partial(R \times I)$ clearly contains $R$ as an embedded proper subsurface.

Now $Y \backslash(R \times I)$ is not necessarily a handlebody (indeed, it will be a handlebody precisely when $R$ is a so-called free Seifert surface). By adding a collection of threedimensional 1-handles $\left\{h_{i}\right\}_{i=1}^{k}$ to $R \times I$ we can ensure that

$$
H_{\alpha}=Y \backslash\left(R \times I \cup h_{1} \cup \cdots \cup h_{k}\right)
$$

is a handlebody (of genus $2 g+k$ ). Without loss of generality, we may assume that the feet of the 1-handles lie on $R \times\{0\}$, so that $R=R \times\{1\} \subset \partial H_{\alpha}$ is still embedded. Let $\beta_{i}$ denote the belt circles of the 1 -handles for $i=2 g+1, \ldots, 2 g+k$.

Finally, pick a collection $\left\{\alpha_{i}\right\}_{i=1}^{2 g+k}$ of linearly independent curves on $\Sigma_{2 g+k}=\partial H_{\alpha}$ which bound disks in $H_{\alpha}$. Then

$$
\left(\Sigma_{2 g+k},\left\{\alpha_{1}, \ldots, \alpha_{2 g+k}\right\},\left\{\beta_{1}, \ldots, \beta_{2 g+k}\right\}\right)
$$

is the desired Heegaard diagram.

Remark 3.1 Note that, by construction, the Heegaard diagram

$$
\left(\Sigma_{2 g+k},\left\{\alpha_{1}, \ldots, \alpha_{2 g+k}\right\},\left\{\beta_{2 g+1}, \ldots, \beta_{2 g+k}\right\}\right)
$$

(ie the Heegaard diagram of the lemma without the first $2 g \quad \beta$ curves) specifies $Y \backslash$ $(R \times I)$.

\subsection{Turning the diagram into a surface diagram}

Having produced a Heegaard diagram for $Y$ containing $R$, we now describe how to turn this into a surface diagram for $R$.

Begin with the Heegaard diagram of Section 3.1. The desired result is achieved through the following algorithm (see Figure 3.1 for a depiction of the algorithm):

(1) Pick two points $\{z, w\} \subset K=\partial R$. The points divide $K$ into two arcs, which we label by $A$ and $B$. Remove disc neighborhoods $\{D(z), D(w)\} \subset \Sigma$ of the two points and call the resulting surface-with-boundary $\Sigma$.

If $A \cap \beta_{i}=\varnothing$ and $B \cap \alpha_{i}=\varnothing$ for all $i$, then we are done. If not, proceed to Step 2 . 
(2) Without loss of generality, assume $p \in A \cap \beta_{i}$ (if $p \in B \cap \alpha_{i}$, the same process applies with the roles of $A$ and $B, \alpha$ and $\beta$ reversed). Remove $p$ by one of the following operations:

- Isotopy Starting from $p$, perform a finger move of $\beta_{i}$ along the $A$ arc until a boundary component of $\Sigma$ is reached. If other $\beta$ curves are encountered, perform the finger move to these curves as well to ensure that no intersections among $\beta$ curves are created. Handleslide $\beta_{i}$, along with any other curves picked up by the isotopy, over the boundary component of $\Sigma$.

- Stabilization Let $A_{0} \subset A$ be the component of $A$ containing $p$. To perform this move, we pick the point $p$ such that no $\beta$-curve intersects at least one component of $A_{0} \backslash\{p\}$. Choose a subarc $\gamma \subset A_{0}$ containing $p$ that satisfies $\gamma \cap \alpha_{i}=\varnothing$ for all $i$. Subdivide $K$ so that $\gamma$ is labeled $B$ and the two arcs adjacent to $\gamma$ are labeled $A$. Let $D(\gamma)$ be a neighborhood of $\gamma$. Adjoin $\partial(D(\gamma))$ to the collection of $\alpha$ curves. Similarly, pick one of the two $A$ arcs adjacent to $\gamma$, and adjoin the boundary of its neighborhood to the $\beta$ curves. Finally, remove neighborhoods of the endpoints of $\gamma$ from $\Sigma$.

(3) If $A \cap \beta_{i}=\varnothing, B \cap \alpha_{i}=\varnothing$ for all $i$ then we are done. If not, repeat Step 2.

Proposition 3.2 The above algorithm terminates at a surface diagram adapted to $R$.

Proof As there are only a finite number of points in the initial set $\left\{A \cap \beta_{i}, B \cap \alpha_{i}\right\}$, it is clear that the above algorithm terminates. To see that we have produced a surface diagram, first observe that the algorithm can be reinterpreted as an algorithm to convert the original diagram into a multipointed Heegaard diagram for $K \subset Y$. Indeed, instead of removing neighborhoods of $\{z, w\}$ and the endpoints of $\gamma$ in Steps 1 and 2, respectively, we could simply keep track of these points as pairs $\left\{z_{i}, w_{i}\right\}$ (and labeling so that the $z$ are always the initial point of some $A$ arc, oriented by the orientation of $K)$. The resulting multipointed Heegaard diagram is then adapted to $K$, in the sense of [19, Definition 2.1]. Generalizing [11, Example 2.4 and Proposition 9.2] to the case of multipointed diagrams shows that removing neighborhoods of the basepoints produces a balanced diagram adapted $Y_{2 n}(K){ }^{1}$

Finally, observe that since the original diagram contained $R$ as a proper subsurface, the terminal diagram contains $R$ as a quasipolygon of the desired form. Indeed, the $A$ and $B$ edges of the quasipolygon are the $A$ and $B$ arcs produced by the algorithm. Strictly speaking, we must make a local modification to these arcs as specified by Figure 3.2 to ensure that $\partial R$ is of the appropriate form.

\footnotetext{
${ }^{1}$ Note that $n$ is the number of stabilizations performed in the algorithm +1 .
} 
(1)
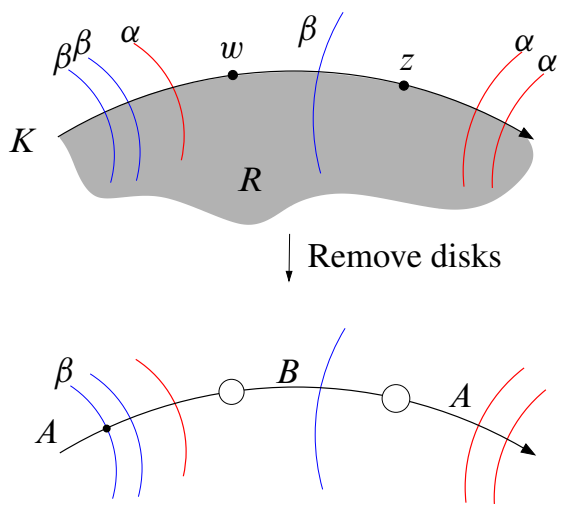

(2)
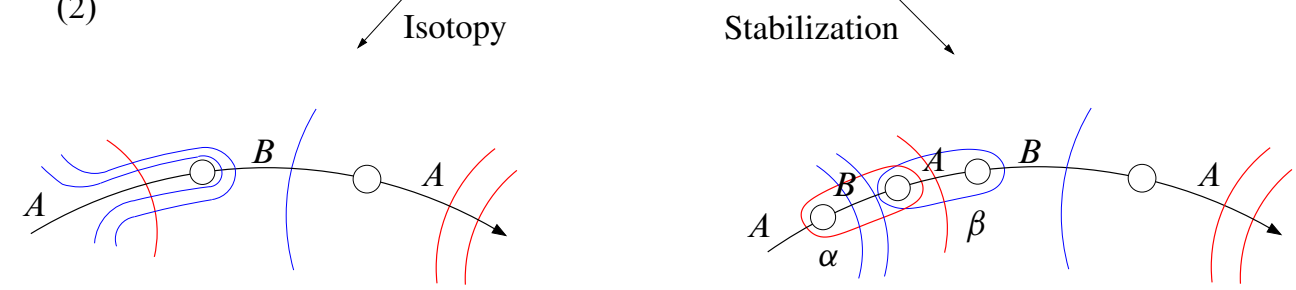

Figure 3.1: Turning a Heegaard diagram for $Y$ containing $R$ into a surface diagram for $R$

\subsection{Explicit Heegaard diagrams for Seifert surfaces in $S^{3}$}

We now describe an explicit diagram adapted to a Seifert surface in $S^{3}$. To begin, we isotope $R$ so that it consists of a disc with $2 g$ bands attached to it. One way to do this is to note that since $R$ is a surface with one boundary component, it is homotopy equivalent to a CW complex with one $0-$ cell and $2 g$-cells. Represent the $0-$ cell by a disk neighborhood $D$ of an interior point. Represent the 1 -cells by $2 g$ disjoint arcs on $R$, each of whose endpoints lie in $\partial D$. Now let $F$ be a regular neighborhood of $D$ and

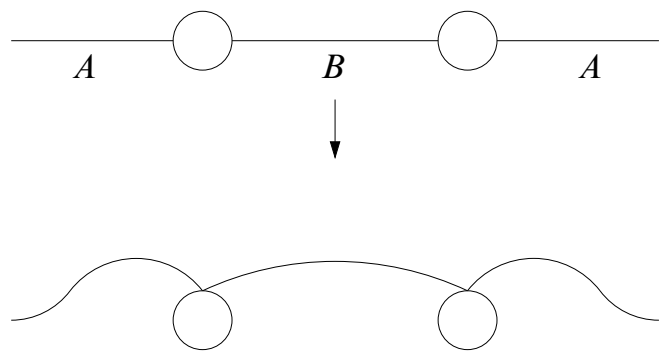

Figure 3.2: Local modifications of the $A$ and $B$ arcs 
the $2 g$ arcs. Then $R$ deformation retracts onto $F$ and $\partial R$ stays an embedded circle in $S$ throughout. This induces an isotopy which takes $(R, K)$ to $(F, \partial F)$. Indeed, if the surface $R$ is presented in some nice fashion then this gives an algorithm to get such a pair $(F, \partial F)$. An example of this construction is shown in Figure 3.3 for the minimal genus Seifert surface of the trefoil.
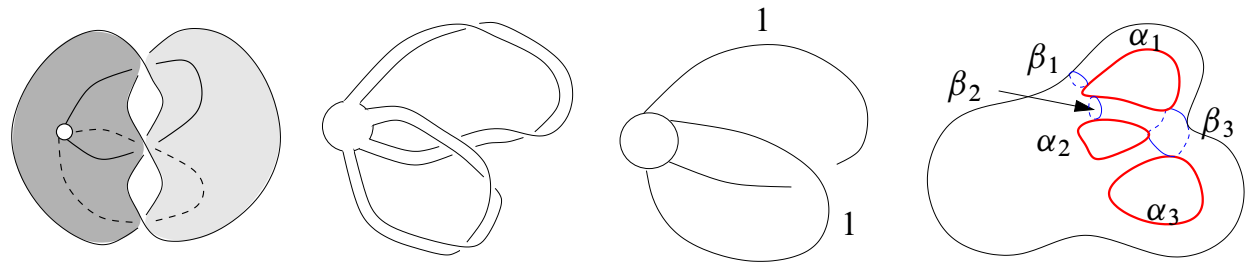

Figure 3.3: Starting from a Seifert surface for the trefoil, we first retract to a neighborhood of a one skeleton possessing a single 0 -cell. We then encode this surface with a planar diagram, where the 1 indicate that the corresponding bands have a full right-handed twist. The third shows the handlebody which results from thickening the diagram. The thick red curves are $\alpha$-curves specifying the complementary handlebody. Each band contributes a thin blue $\beta$ curve. The final $\beta$ curve comes from the crossing in the diagram, according to Figure 3.4 below.

Assuming, then, that $R$ is represented as above, proceed by contracting each band of $R$ to an arc. We may assume that the resulting disk with $2 g$ arcs lies in a subset homeomorphic to $\mathbb{R}^{3}$ and, moreover, that there exists a plane $\mathbb{R}^{2} \subset \mathbb{R}^{3}$ onto which projection yields a planar diagram satisfying:

- The disc ( 0 -handle) of $R$ is embedded in $\mathbb{R}^{2}$ and no arc is projected to its interior.

- The arcs have only finitely many transverse double points.

Keeping track of the crossing information at the double points, together with the framing of the band corresponding to each arc, ${ }^{2}$ we obtain a planar diagram from which we can recover the surface, up to isotopy. Let $k$ be the number of double points (crossings) in this planar diagram.

Proceed by thickening the diagram in $\mathbb{R}^{2}$ to obtain a handlebody, $H_{\beta}$, in $\mathbb{R}^{3}$. The genus of $H_{\beta}$ is $2 g+k$, and we let $\Sigma=\partial H_{\beta}$ denote its boundary. Intersecting $\Sigma$ with the original plane results in $(2 g+k+1)$ circles. Choose any $(2 g+k)$ of these to be the $\alpha$ circles. Clearly, $\left(\Sigma, \alpha_{1}, \ldots, \alpha_{2 g+k}\right)$ represents the complement of $H_{\beta}$.

\footnotetext{
${ }^{2}$ Each band $b$ comes with a framing which is \#\{full right-handed twists of $\left.b\right\}-$ \#\{full left-handed twists of $b\}$.
} 
Corresponding to each of the $2 g$ arcs we obtain a $\beta$ circle. This circle is the boundary of a disk which intersects the arc in a point close to the disc $D$. Label these circles, $\beta_{i}$ for $i=1, \ldots, 2 g$. Finally, to each crossing in the planar diagram we add a $\beta$ circle according to the convention of Figure 3.4. These circles are labeled $\beta_{2 g+1}, \ldots \beta_{2 g+k}$.
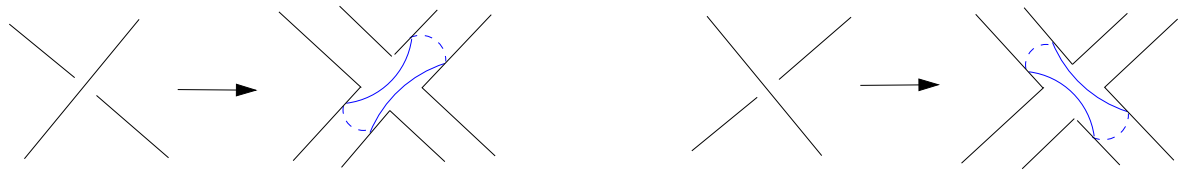

Figure 3.4: Adding $\beta$ curves for crossings

The resulting diagram

$$
\left(\Sigma,\left\{\alpha_{1}, \ldots, \alpha_{2 g+k}\right\},\left\{\beta_{1}, \ldots, \beta_{2 g+k}\right\}\right)
$$

represents $S^{3}$ and contains $R$ by construction. An example of such a Heegaard diagram for the case of the trefoil is shown in Figure 3.3.

Next, we remove $8 g$ discs from the Heegaard diagram while simultaneously adding $4 g-1$ pairs of $\alpha$ and $\beta$ circles to adapt the diagram to $R$.

To describe this, first observe that the Heegaard surface can be divided into two parts; the handles and the (punctured) sphere. The handles arise from the boundary of a regular neighborhood of the arcs in the planar diagram, while the sphere comes from the boundary of a regular neighborhood of the disc $D$. Next, note that $K=\partial R$ is naturally embedded in $\Sigma$ in such a way that it does not intersect the circles $\beta_{2 g+1}, \ldots, \beta_{2 g+k}$, and intersects each of the other $\beta$ circles exactly twice. See Figure 3.5

Up to isotopy in $\Sigma$, we can assume that $K$ consists of two parallel strands in each handle. We can further assume that $K$ lies mostly in the top part of $\Sigma$ (namely, the part of $\Sigma$ lying above the plane where the diagram of $R$ was embedded). It passes to the bottom of $\Sigma$ only in the handles to account for the framing and crossing information of the bands. For each of the $4 g$ intersection points $\left\{p_{2 i-1}, p_{2 i}\right\} \in K \cap \beta_{i}$ for $i=1, \ldots, 2 g$, we remove two small discs from $\Sigma$ next to $p$, one on either side of $\beta_{i}$. We denote the new surface-with-boundary by $\Sigma^{\prime}$. Removing the discs separates $K$ into $8 g$ arcs, $\left\{A_{j}, B_{j}\right\}, j=1, \ldots, 4 g$, with $p_{j} \in B_{j}$. For each $j \neq 4 g$, we add a curve, $\alpha_{2 g+k+j}$, which encircles $B_{j}$ together with the boundary components of $\Sigma$ created by removing the discs near the endpoints of $B_{j}$. Similarly, for each $j \neq 4 g$ we add a curve, $\beta_{2 g+k+j}$, which encircles $A_{j}$ and the boundary components of $\Sigma$ created by removing the discs near the endpoints of $A_{j}$. 


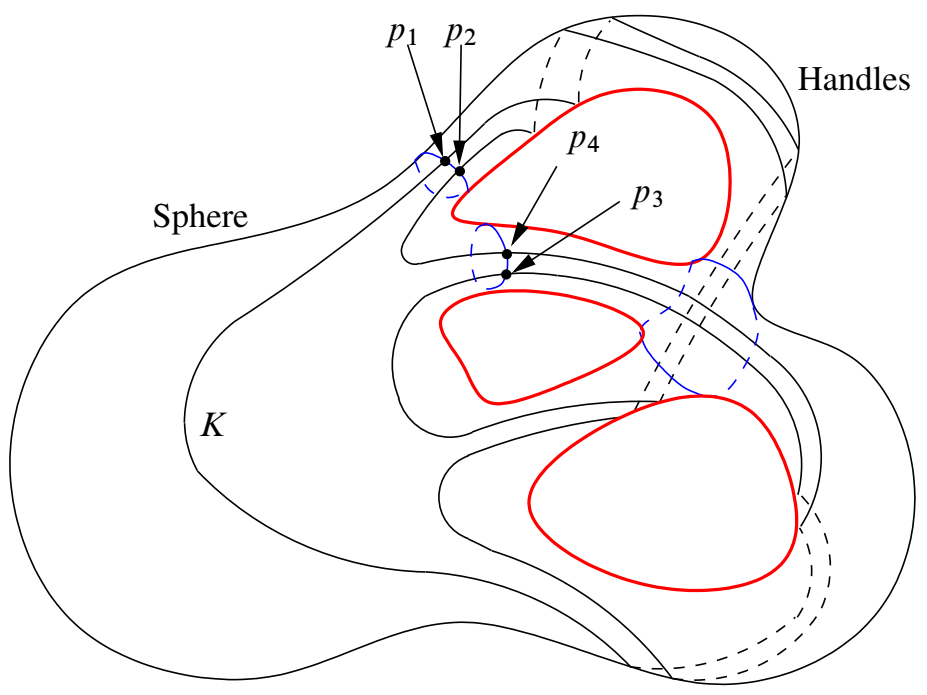

Figure 3.5: The embedding of $K=\partial R$ in $\Sigma$

Since $\Sigma$ contained $R$ before the discs were removed, it is straightforward to construct a quasipolygon, $P \subset \Sigma^{\prime}$, which satisfies the requirements of Definition 2.13. Indeed, the $A$ and $B$ edges of $P$ are isotopic to the $A$ and $B$ arcs of the previous paragraph, as in Figure 3.2. We have arrived at a surface diagram for $R$ :

$$
\left(\Sigma^{\prime},\left\{\alpha_{1}, \ldots, \alpha_{6 g+k-1}\right\},\left\{\beta_{1}, \ldots, \beta_{6 g+k-1}\right\}, P\right) .
$$

See Figure 3.6 for the diagram adapted to the Seifert surface of the trefoil.

It is now easy to obtain a balanced diagram for the sutured manifold complementary to $R$ : Delete the interior of $P$ from the Heegaard diagram and take two copies of the subsurface. Delete all the $\alpha$ arcs in one of the copies and identify its $B$ arcs with the corresponding $B$ arcs in the Heegaard diagram. Similarly, delete all the $\beta$ arcs in the other copy, and identify all its $A$ arcs with the corresponding $A$ arcs in the Heegaard diagram. The process is shown locally in the first part of Figure 3.7. The resulting balanced diagram represents $S^{3}(R)$. The Heegaard surface has genus $(7 g+k-1)$ and 1 boundary component, and has $(6 g+k-1) \alpha$ circles and $\beta$ circles each. The final sutured diagram for the trefoil example is shown in Figure 3.7.

Remark 3.3 The diagram above is a special case of the general construction discussed in the previous two subsections. The procedure by which we handled the crossing regions of the planar diagram associated to $R$ is equivalent to adding 1 -handles to $R \times I$ to make the complement into a handlebody. See Figure 3.8. Removing discs and 
adding $\alpha / \beta$ pairs is simply a specific implementation of the algorithm from Section 3.2. Indeed, the diagram of this subsection can be seen as extremal: at every step in the algorithm we used a stabilization. The other extremal case, where we use only isotopies, will be implemented in Section 4 to calculate the sutured Floer homology for the Seifert surfaces of $8_{3}$.
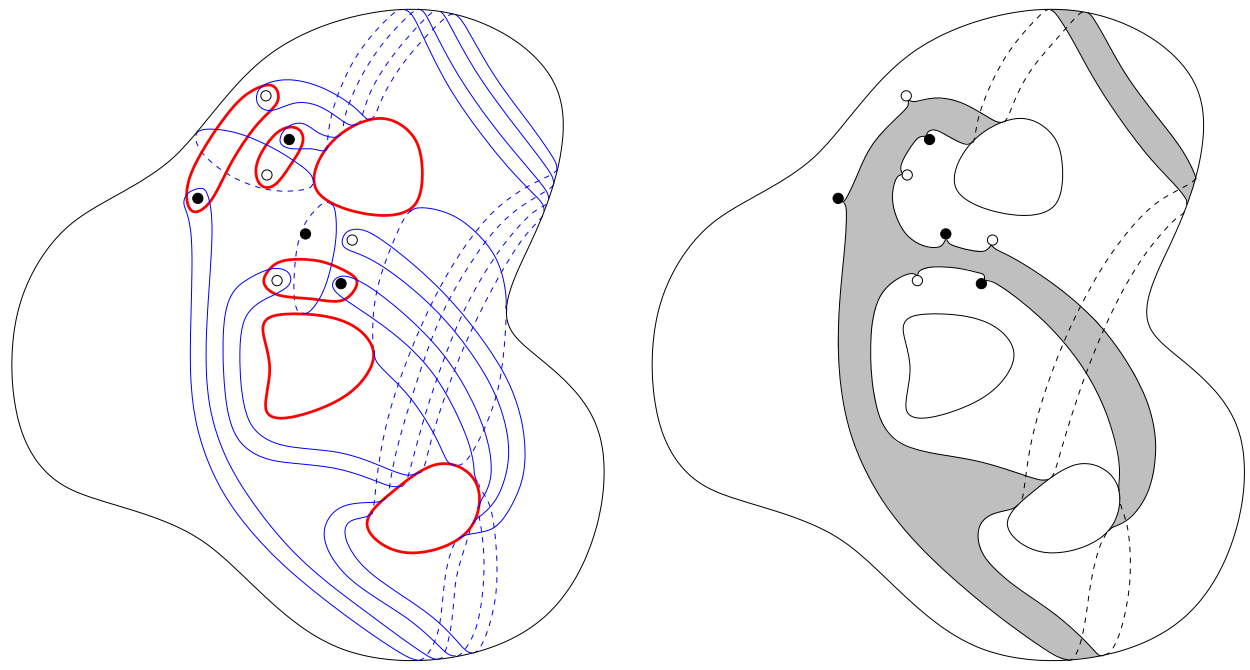

Figure 3.6: The Heegaard diagram adapted to the Seifert surface of the trefoil
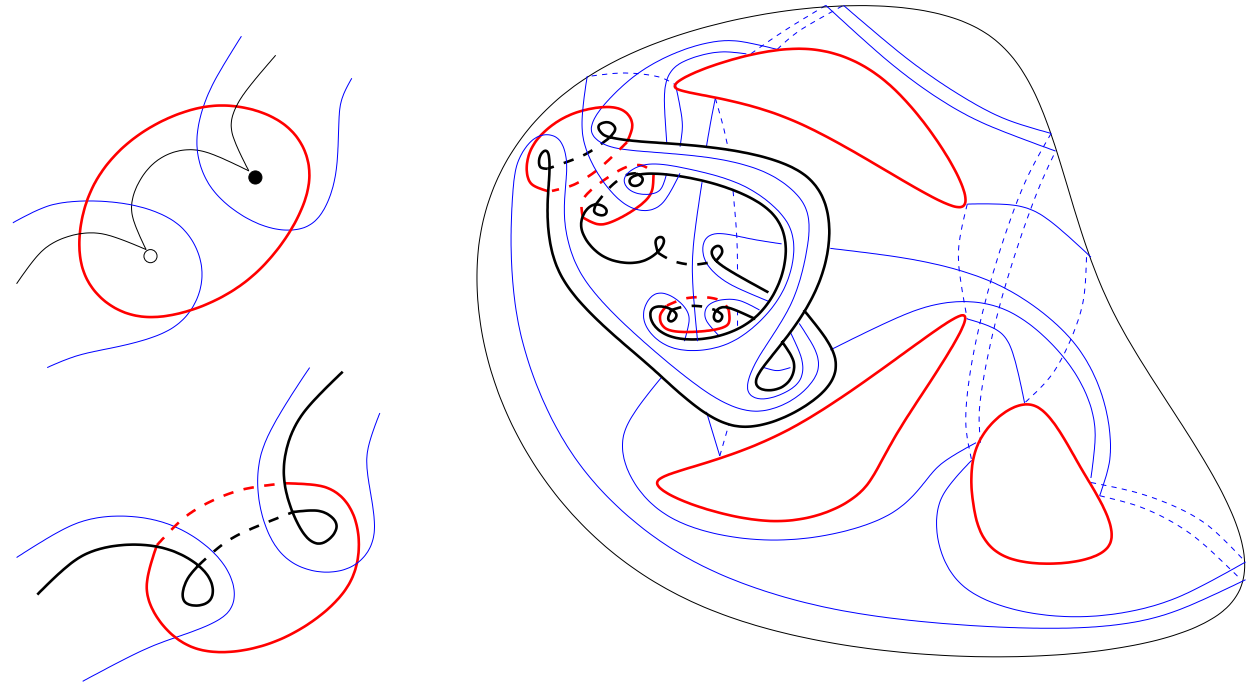

Figure 3.7: The sutured diagram for the complement of the Seifert surface of the trefoil 


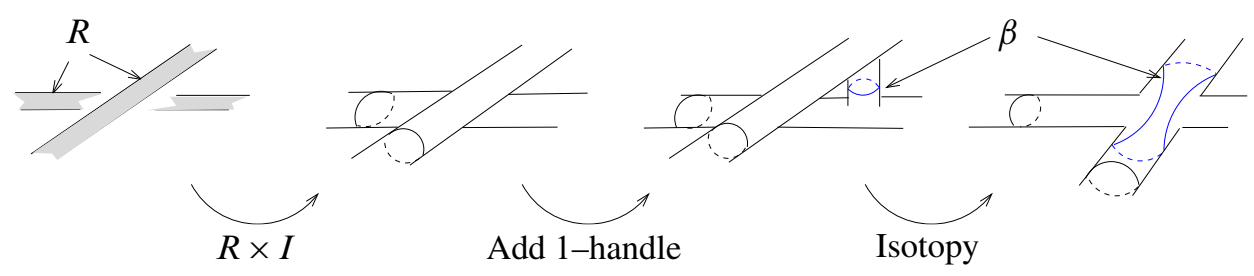

Figure 3.8: Adding handles to make the complement into a handlebody

Remark 3.4 The diagram above is usually not the minimal genus possible. Picking a different set of 1 -handles to add to $R \times I$ will frequently lower the genus of the Heegaard diagram significantly. The minimal number of 1-handles necessary to add to an embedded handlebody, $H_{g}$, so that the complement is a handlebody is often referred to as the tunnel number of $H_{g}$.

While we do not use this diagram for the computation in the next section, it may be of future use to note that the combinatorics of the diagram enable one to calculate $\operatorname{SFH}\left(S^{3}(R)\right)$ without decomposing the diagram. More precisely, recall from the discussion surrounding Theorem 2.19 that the generators of the chain complex associated to the decomposed diagram are in bijection with the outer generators $C\left(\mathcal{O}_{P}\right)$ on the surface diagram. Indeed, Theorem 2.19 shows that the outer generators form a subcomplex of $\operatorname{SFH}\left(S_{2 n}^{3}(K)\right)$ whose homology is isomorphic to $\operatorname{SFH}\left(S^{3}(R)\right)$. However, it is not clear that the obvious bijection between generators of $C\left(\mathcal{O}_{P}\right)$ and $\operatorname{SFH}\left(S^{3}(R)\right)$ induces the isomorphism on homology. The differentials on these two complexes could be quite different, as their definition is in terms of quite different Heegaard diagrams. It is only after altering the surface diagram severely to make it "nice" that an identification between the differentials is established. In light of this, it is nice to know that we can compute the homology of $\operatorname{SFH}\left(S^{3}(R)\right)$, as a relatively $H_{1}\left(S^{3} \backslash R\right)$ graded group, without decomposing the surface diagram. Indeed we have the following proposition:

Proposition 3.5 Let $\mathcal{H}=(\Sigma, \boldsymbol{\alpha}, \boldsymbol{\beta}, P)$ be the explicit surface diagram for a Seifert surface $R \subset S^{3}$ described above, and let $\mathcal{H}^{\prime}=\left(\Sigma^{\prime}, \boldsymbol{\alpha}^{\prime}, \boldsymbol{\beta}^{\prime}\right)$ be the decomposed diagram. Denote the associated chain complexes by $(C(\mathcal{H}), \partial)$ and $\left(C\left(\mathcal{H}^{\prime}\right), \partial^{\prime}\right)$. Then the differential on the subcomplex $C\left(\mathcal{O}_{P}\right) \subset C(\mathcal{H})$ generated by outer intersection points is equal to that on $C\left(\mathcal{H}^{\prime}\right)$, under the obvious isomorphism of chain groups induced by the bijection of generators. In particular, the relative $\operatorname{Spin}^{c}$-grading on $\operatorname{SFH}\left(S^{3}(R)\right)$ can be computed by considering the difference $\epsilon(\boldsymbol{x}, \boldsymbol{y})$ of two generators $\boldsymbol{x}, \boldsymbol{y} \in C\left(\mathcal{O}_{P}\right)$ as a 1 -cycle in $H_{1}\left(S^{3} \backslash R\right)$.

Remark 3.6 For more details on how to regard the difference $\epsilon(\boldsymbol{x}, \boldsymbol{y})$ of outer generators as an element in $H_{1}\left(S^{3} \backslash R\right)$, see Section 4.3.2 below. 
Proof Let $\boldsymbol{x}, \boldsymbol{y} \in C\left(\mathcal{O}_{P}\right) \subset C(\mathcal{H})$ denote outer generators; that is, generators whose intersection points lie outside the quasipolygon $P$ representing $R$. Let $\boldsymbol{x}^{\prime}, \boldsymbol{y}^{\prime} \in C\left(\mathcal{H}^{\prime}\right)$ denote the corresponding generators for the decomposed diagram. It suffices to show that

$$
\boldsymbol{y} \in \partial \boldsymbol{x} \quad \text { if and only if } \boldsymbol{y}^{\prime} \in \partial^{\prime} \boldsymbol{x}^{\prime}
$$

(recall that we are working with $\mathbb{Z} / 2 \mathbb{Z}$ coefficients). Let $\phi \in \pi_{2}(\boldsymbol{x}, \boldsymbol{y})$ be a domain connecting the outer generators. Proving (1) will be accomplished by showing that $\widehat{\mathcal{M}}(\phi) \neq \varnothing$ implies $\phi \cap\{B$-arcs $\}=\varnothing$. In other words, the domains which contribute to $\partial$ do not intersect the $B$ arcs on the quasipolygon. To see why this implies (1), note that any $\phi$ satisfying $\phi \cap\{B$-arcs $\}=\varnothing$ can be thought of as $\phi^{\prime} \in \pi_{2}\left(\boldsymbol{x}^{\prime}, \boldsymbol{y}^{\prime}\right)$, actually such domains are in one to one correspondence with domains on the decomposed diagram that support holomorphic representatives. Indeed, if $\phi^{\prime}$ is a domain connecting $\boldsymbol{x}^{\prime}$ and $\boldsymbol{y}^{\prime}$ that has a holomorphic representative, then projecting this holomorphic map to $\Sigma$ we see that $\phi=p\left(\phi^{\prime}\right)$ also has a holomorphic representative, thus $\phi \cap\{B-\operatorname{arcs}\}=\varnothing$. Furthermore, for such domains, an almost complex structure on $\Sigma \times D^{2}$ achieving transversality for $\mathcal{M}(\phi)$ can be extended to an almost complex structure (under the embedding of $\Sigma \backslash B \subset \Sigma^{\prime}$ away from the sutures) on $\Sigma^{\prime} \times D^{2}$ which achieves transversality for $\mathcal{M}\left(\phi^{\prime}\right)$ (here we are thinking in terms of the cylindrical version of Floer homology [17]). In this way we see that if $\phi \cap\{B-\operatorname{arcs}\}=\varnothing$, then $\widehat{\mathcal{M}}(\phi) \neq \varnothing$ if and only if $\widehat{\mathcal{M}}\left(\phi^{\prime}\right) \neq \varnothing$. Examining each $\phi$, we obtain (1).

Thus it suffices to show that $\widehat{\mathcal{M}}(\phi) \neq \varnothing$ implies $\phi \cap\{B$-arcs $\}=\varnothing$. By contradiction, suppose that $\widehat{\mathcal{M}}(\phi) \neq \varnothing$ and $\phi \cap\{B-\operatorname{arcs}\} \neq \varnothing$ for some domain $\phi$. In Figure 3.9, we have marked various components of $\mathcal{H} \backslash(\boldsymbol{\alpha} \cup \boldsymbol{\beta})$. The quasipolygon $P$ is shaded. As usual, the thick red circles are $\alpha$ circles, and the thin blue circles are $\beta$ circles. Let $n_{p}$ be the multiplicity of $\phi$ in a region marked $p$. Recall from Lemma 2.15 that if $\widehat{\mathcal{M}}(\phi) \neq \varnothing$, then $n_{p} \geq 0$ for all $p \in \Sigma$.

Our first observation deals with the parts of the diagram that appear locally like the lower left part of Figure 3.9. We claim that for $\boldsymbol{x}, \boldsymbol{y} \in \mathcal{O}_{P}$, all domains satisfy $n_{k}-n_{i}=n_{l}-n_{j}$. This follows from the fact that $\boldsymbol{x}, \boldsymbol{y} \in \mathcal{O}_{P}$ implies there are no points of $\boldsymbol{x}, \boldsymbol{y} \in P$, and hence there are no corner points of $\phi$ contained in $P$.

Next, we deal with the arcs in $\phi$ that hit the $B$ arc shown in the first part of that figure. Label the regions in the handle part of $\mathcal{H}$ by $a, b, c$ and $g$ and let the regions in the sphere part of $\mathcal{H}$ be $d, e, f$ and $h$. There are two cases.

- $n_{d} \neq 0$. Since $n_{e}=0$ ( $e$ contains a suture) and $\boldsymbol{x}$ and $\boldsymbol{y}$ contain no points in $P$, we see that $n_{h}-n_{f}=n_{d}-n_{e}>0$. Thus $n_{h}>0$ and since $h$ is in the spherical part of 
$\mathcal{H}$, the region marked $h$ is the same region as the disk region of $R$, marked $m$ in the third part of Figure 3.9. But the disk part contains a suture, leading to a contradiction.

- $n_{c} \neq 0$. Again, since $n_{b}=0$, a similar argument shows that $n_{g}-n_{a}>0$. Proceed by examining the multiplicities of $\phi$ in the regions of the handle part of $\mathcal{H}$ which border the $\beta$ curve that separated $a$ from $g$. Of course, there may be $\alpha$ curves encountered on the way, as shown in the lower left part of Figure 3.9. However, our first observation above shows that the difference of the multiplicities of $\phi$ on the two regions adjacent to the $\beta$ curve stays positive. Proceeding along the handle until we reach the disk region, we again get $n_{m}>0$. Thus in either case, we are done.
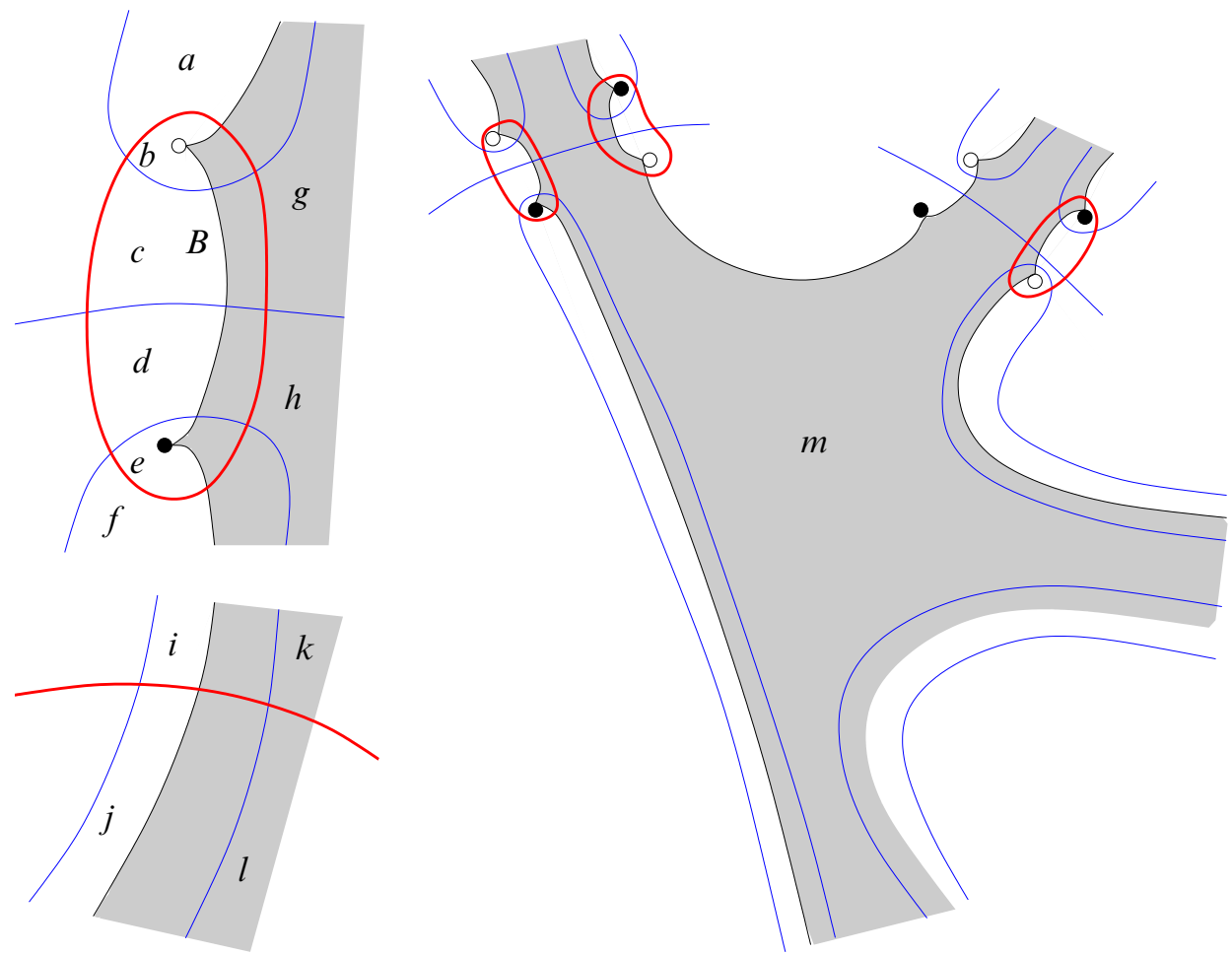

Figure 3.9: Local coefficients of $\phi$ at various points

\section{Using $\operatorname{SFH}(Y(R))$ to distinguish Seifert surfaces}

Given an oriented knot $K \subset S^{3}$, there are several notions of equivalence one could consider for its Seifert surfaces. We will consider two Seifert surfaces, $R_{1}, R_{2}$, to be 
equivalent if there is an isotopy of $S^{3}$ taking $R_{1}$ to $R_{2}$. Note that this is the same as considering $R_{1}$ and $R_{2}$ to be equivalent if there is an orientation preserving diffeomorphism between the pairs $\left(S^{3}, R_{1}\right)$ and $\left(S^{3}, R_{2}\right)$ (since the group of orientation preserving diffeomorphisms of the three-sphere is path-connected). A more restrictive notion, called strong equivalence, regards $R_{1}$ and $R_{2}$ as equivalent if they are isotopic in the complement of $K$. Note that we can discuss whether the surfaces $R_{1}$ and $R_{2}$ are equivalent if $\partial R_{1}$ and $\partial R_{2}$ are equivalent knots, while we can ask whether two surfaces are strongly equivalent only if $\partial R_{1}=\partial R_{2}$.

The remainder of this section will be devoted to showing how the sutured Floer homology invariants of $S^{3}(R)$ can be used to distinguish nonequivalent Seifert surfaces. We do this through a detailed discussion of an example. Figure 4.1 depicts two Seifert surfaces, $R_{1}$ and $R_{2}$, each bounded by the knot $8_{3}$. Note that $R_{1}$ is obtained by plumbing two bands with +2 and -2 full twists, respectively, and $R_{2}$ is obtained by taking the dual plumbing. So indeed the two surfaces bound the same oriented knot. We will show that $R_{1}$ and $R_{2}$ are inequivalent. Combining this with the results of [15], it will follow that these represent all isotopy classes of Seifert surface for $8_{3}$ (see Proposition 4.3 below).
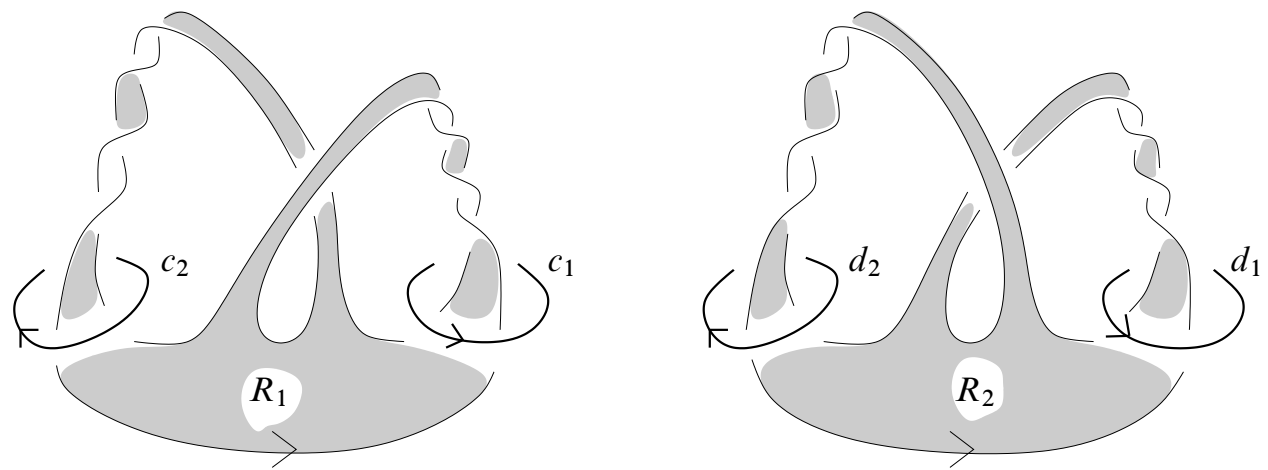

Figure 4.1: Two different Seifert surfaces for the same knot, $8_{3}$. The outward normal to each surface on the shaded region is out of the plane of the page (towards the reader).

\subsection{Classical methods}

Before beginning, we make some preliminary remarks regarding this particular example and the applicability of previously known techniques. One effective way to distinguish isotopy classes of surfaces is through the fundamental group of their complements; 
see [1]. In the present case, however, this obviously fails. Indeed, $S^{3} \backslash R_{1}$ is homeomorphic to $S^{3} \backslash R_{2}$; they are both open handlebodies of genus 2 . Thus any attempt to use the fundamental group will be fruitless.

Even in the case when the complements of the surfaces are homeomorphic, classical techniques could still be of use. The Seifert form provides a useful obstruction to finding an isotopy between two surfaces. To describe this, recall that $H_{1}(R ; \mathbb{Z})$ is equipped with a bilinear form,

$$
Q_{R}: H_{1}(R ; \mathbb{Z}) \otimes H_{1}(R ; \mathbb{Z}) \longrightarrow \mathbb{Z},
$$

called the Seifert form. Given curves $a$ and $b$ in $R$, let $b^{+}$be a push-off of $b$ in the direction specified by the positive unit normal vector field of $R$. Then the Seifert form evaluated on $([a],[b])$ is the linking number of $a$ with $b^{+}$in $S^{3}$. Suppose now that two Seifert surfaces are isotopic. It follows that they have congruent Seifert forms. This means that there exists $W \in S L(2 g, \mathbb{Z})$ for which

$$
V_{R_{2}}=W^{T} V_{R_{1}} W
$$

where $V_{R_{i}}$ are integral matrices representing $Q_{R_{i}}$ with respect to given bases for $H_{1}\left(R_{i} ; \mathbb{Z}\right) \cong \mathbb{Z}^{2 g}$. Concretely, $W$ is the matrix representing the isomorphism of $H_{1}(R ; \mathbb{Z})$ induced by the diffeomorphism $\left(S^{3}, R_{1}\right) \cong\left(S^{3}, R_{2}\right)$. Thus, to show that two Seifert surfaces are inequivalent, it suffices to show that their Seifert forms are not congruent (see [31] for applications of this method to Seifert surfaces of some pretzel knots). In the situation at hand, however, this method also fails. For the obvious symplectic bases, the following matrices represent the Seifert forms of $R_{1}$ and $R_{2}$ :

$$
V_{R_{1}}=\left(\begin{array}{rr}
2 & 0 \\
1 & -2
\end{array}\right) \quad \text { and } \quad V_{R_{2}}=\left(\begin{array}{ll}
2 & -1 \\
0 & -2
\end{array}\right)
$$

The intersection forms in the same basis are represented by

$$
U_{R_{1}}=U_{R_{2}}=\left(\begin{array}{rr}
0 & 1 \\
-1 & 0
\end{array}\right)
$$

One can easily check, however, that $V_{R_{1}}$ and $V_{R_{2}}$ are congruent. An appropriate element of $S L(2, \mathbb{Z})$ is

$$
W=\left(\begin{array}{rr}
4 & -5 \\
-3 & 4
\end{array}\right)
$$

Note that $W$ also preserves the standard symplectic form on $\mathbb{Z}^{2}$, ie $U_{R_{2}}=W^{T} U_{R_{1}} W$, so the Seifert form and the intersection form together are incapable of distinguishing $R_{1}$ and $R_{2}$. 
Finally, we remark that techniques from the theory of sutured manifolds have been quite fruitful in studying Seifert surfaces up to strong equivalence. See, for instance, Kakimizu [15] and Kobayashi [16]. Indeed Kakimizu [15] has used these techniques to classify minimal genus Seifert surfaces up to strong equivalence for knots of 10 or fewer crossings. In particular, it follows that if $R_{1}$ and $R_{2}$ are dual plumbings of a +2 and a -2 twisted band, then they represent distinct strong equivalence classes, and that these are the only two such classes. It should be noted, however, that equivalence and strong equivalence are quite different. For instance, if the bands both had framing +2 , then the boundary of the resulting dual surfaces would each be the knot 74 . It follows from [15] that these two surfaces are strongly inequivalent. It is easy to verify, however, that they are isotopic, and hence equivalent in our sense. As our techniques are able to distinguish surfaces up to isotopy, we will make no further reference to strong equivalence.

It is worth mentioning that not only $S^{3} \backslash R_{1}$ and $S^{3} \backslash R_{2}$, but even the sutured manifolds $S^{3}\left(R_{1}\right)$ and $S^{3}\left(R_{2}\right)$ are diffeomorphic. Indeed, let $A$ and $A^{\prime}$ be +2 and -2 twisted bands in $S^{3}$, respectively. The complementary sutured manifold $S^{3}\left(A^{\prime}\right)$ is $S^{1} \times D^{2}$ with sutures the torus link $T(4,-2)$. A positive Dehn twist along $\{\mathrm{pt}\} \times D^{2}$ maps $T_{4,-2}$ to $T_{4,-2+4}=T_{4,2}$, so we get $S^{3}(A)$. Now $R_{1}$ is the plumbing of $A$ and $A^{\prime}$, and performing the Dehn twist in the complement of $A^{\prime}$ transforms $S^{3}\left(R_{1}\right)$ into $S^{3}\left(F_{1}\right)$, where $F_{1}$ is the plumbing of two copies of $A$. Similarly, $R_{2}$ is the dual plumbing of $A$ and $A^{\prime}$, so a Dehn twist maps $S^{3}\left(R_{2}\right)$ to $S^{3}\left(F_{2}\right)$, where $F_{2}$ is the dual plumbing of two copies of $A$. We mentioned above that $F_{1}$ and $F_{2}$ are even strongly equivalent, so $S^{3}\left(F_{1}\right)$ and $S^{3}\left(F_{2}\right)$ are diffeomorphic, proving that $S^{3}\left(R_{1}\right)$ and $S^{3}\left(R_{2}\right)$ are also diffeomorphic. Note that if $R_{1}$ and $R_{2}$ were dual plumbings of a $+k$ and a $-k$ twisted band for $k>2$, then the sutured manifolds $S^{3}\left(R_{1}\right)$ and $S^{3}\left(R_{2}\right)$ would not be diffeomorphic. Indeed, $l$ Dehn twists map the torus link $T_{2 k, 2}$ to $T_{2 k, 2+2 k l}$, and $2+2 k l \neq-2$ for $k>2$.

\subsection{The technique}

Suppose that two surfaces $R_{1}$ and $R_{2}$ are isotopic. It follows that the complementary sutured manifolds $S^{3}\left(R_{1}\right)$ and $S^{3}\left(R_{2}\right)$ will be equivalent. Thus to show that $R_{1}$ and $R_{2}$ are inequivalent, it suffices to show that the sutured Floer homology groups $\operatorname{SFH}\left(S^{3}\left(R_{1}\right)\right)$ and $\operatorname{SFH}\left(S^{3}\left(R_{2}\right)\right)$ are different.

The algorithm from the previous section tells us how to construct Heegaard diagrams adapted to the surfaces. From these diagrams, we can identify generators for the chain complexes and determine the difference between the relative $\operatorname{Spin}^{c}$-structures associated to generators $\boldsymbol{x}$ and $\boldsymbol{y}$. 
After analyzing the chain groups, we will determine their homology indirectly through consideration of the Euler characteristic. The total rank of the groups will agree for $R_{1}$ and $R_{2}$, since it equals the rank of the top group of the knot Floer homology of $\partial R_{i}=8_{3}$. Thus the heart of the argument is to distinguish the groups by showing that their $\operatorname{Spin}^{c}$-gradings are different.

Given $\mathfrak{s}_{1}, \mathfrak{s}_{2} \in \operatorname{Spin}^{c}\left(S^{3}\left(R_{i}\right)\right)$ which support nontrivial Floer groups, our analysis of the chain complexes will produce a geometric representative for the difference class $\mathrm{PD}\left[\mathfrak{s}_{1}-\mathfrak{s}_{2}\right] \in H_{1}\left(S^{3} \backslash R_{i} ; \mathbb{Z}\right)$. To show that the Floer homology groups of $S^{3}\left(R_{1}\right)$ and $S^{3}\left(R_{2}\right)$ are different, we thus need a way to distinguish the various difference classes in $H_{1}\left(S^{3} \backslash R_{1} ; \mathbb{Z}\right)$ from those in $H_{1}\left(S^{3} \backslash R_{2} ; \mathbb{Z}\right)$. This is rather subtle, however, since one can have orientation preserving homeomorphisms from $\left(S^{3}, R_{1}\right)$ to $\left(S^{3}, R_{2}\right)$ that induce different isomorphisms from $H_{1}\left(S^{3} \backslash R_{1} ; \mathbb{Z}\right)$ to $H_{1}\left(S^{3} \backslash R_{2} ; \mathbb{Z}\right)$. To remove this ambiguity, we use the Seifert form.

Let $R$ be a Seifert surface for a knot $K \subset S^{3}$, and let $M=S^{3} \backslash \operatorname{Int}(R \times I)$ be the complement of a regular neighborhood of $R$. Then we have the following natural isomorphisms:

$$
\begin{aligned}
H_{1}(R) & \cong H_{1}(R, \partial R) \\
& \cong H^{1}(R) \\
& \cong H^{2}\left(S^{3}, R\right) \\
& \cong H^{2}(M, \partial M) \\
& \cong H_{1}(M)
\end{aligned}
$$

(long exact sequence for the pair)

(Poincaré duality)

(long exact sequence for the pair)

(excision)

(Poincaré duality)

Since the Seifert form is invariant under isotopy of $R$, the above isomorphisms endow $H_{1}(M) \cong H_{1}\left(S^{3} \backslash R\right)$ with a bilinear form which we also denote by $Q_{R}$. Given $a, b \in H_{1}\left(S^{3} \backslash R ; \mathbb{Z}\right)$, let us denote $Q_{R}(a, b)$ by $a \cdot b$. Similarly, using the above isomorphisms, we can endow $H_{1}\left(S^{3} \backslash R\right)$ with another bilinear form which is obtained from the intersection pairing on $H_{1}(R)$. Its value on the pair $(a, b)$ will be denoted by $a \cap b$. The discussion shows that if $h:\left(S^{3}, R_{1}\right) \rightarrow\left(S^{3}, R_{2}\right)$ is an orientation preserving homeomorphism then $h_{*}: H_{1}\left(S^{3} \backslash R_{1}\right) \rightarrow H_{1}\left(S^{3} \backslash R_{2}\right)$ satisfies $a \cdot b=h_{*}(a) \cdot h_{*}(b)$ and $a \cap b=h_{*}(a) \cap h_{*}(b)$.

Let $\left\langle c_{1}, c_{2}\right\rangle$ and $\left\langle d_{1}, d_{2}\right\rangle$ be bases of $H_{1}\left(S^{3} \backslash R_{1}\right)$ and $H_{1}\left(S^{3} \backslash R_{2}\right)$, respectively, as shown on Figure 4.1. Tracing through the isomorphisms in our particular examples shows that matrix representations for $Q_{R_{i}}$ are also given by the matrices $V_{R_{i}}$ above. Thus $c_{i} \cdot c_{j}$ (respectively $d_{i} \cdot d_{j}$ ) is given by the $i j$-th entry of $V_{R_{i}}$. The values of $a \cdot b$ will distinguish the difference classes discussed above which, in turn, will distinguish the sutured Floer homology as relatively graded groups. 

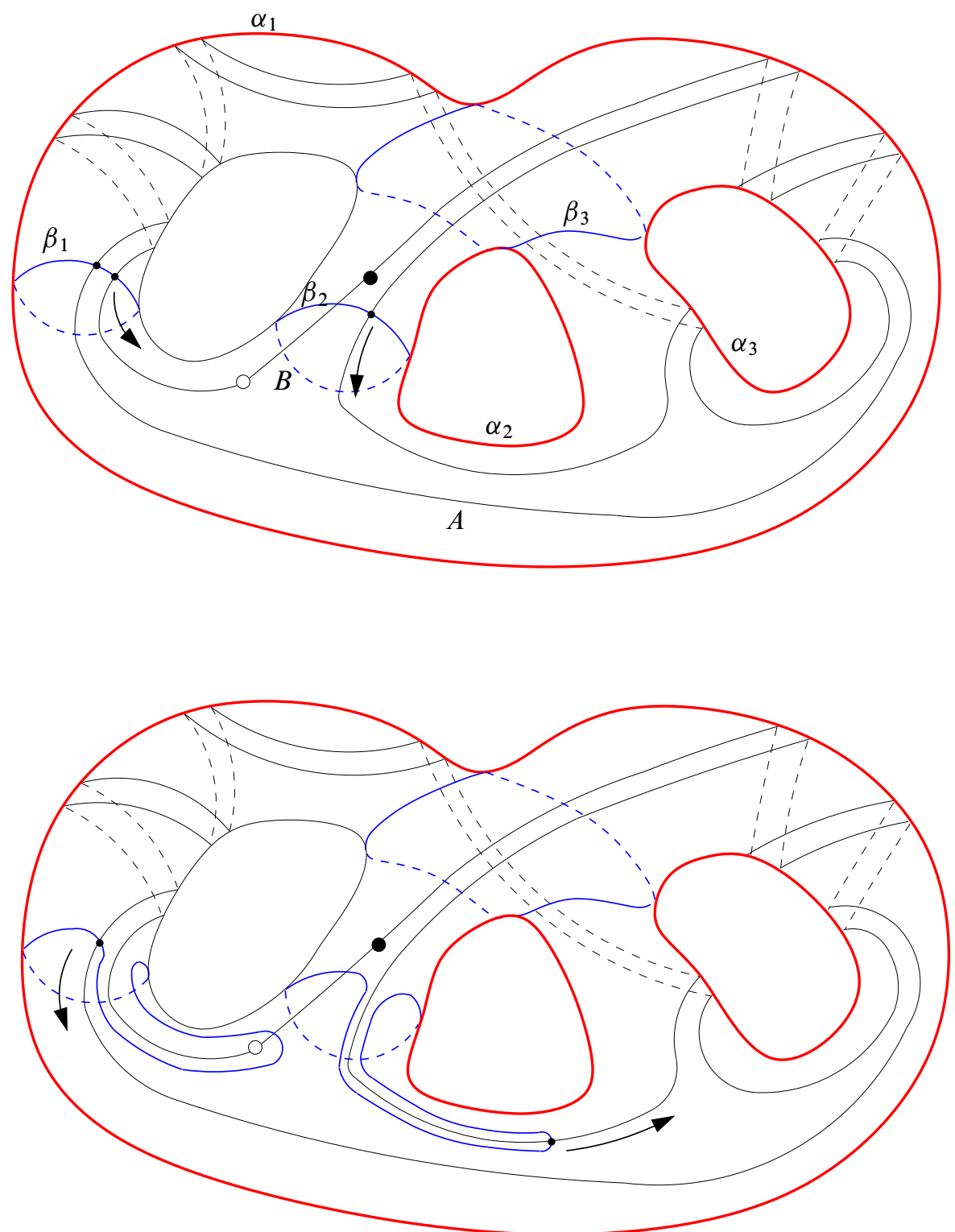

Figure 4.2: Constructing the surface diagram for $R_{1}$. The first figure shows a Heegaard diagram for $S^{3}$ containing $R_{1}$ as a proper subsurface. The thick red curves are $\alpha_{i}, i=1,2,3$ while the thin blue curves are $\beta_{i}, i=1,2,3$. There are three intersection points between the $A$ arc and the $\beta$ curves, which we remove by a sequence of isotopies. 


\subsection{Calculation}

Consider the two Seifert surfaces $R_{1}, R_{2}$ for $8_{3}$ shown in Figure 4.1. In this section, we calculate the sutured Floer homology groups of $S^{3}\left(R_{i}\right)$, showing that there is no isomorphism between the graded groups $\operatorname{SFH}\left(S^{3}\left(R_{1}\right)\right)$ and $\operatorname{SFH}\left(S^{3}\left(R_{2}\right)\right)$ that also preserves the Seifert form. We discuss $R_{1}$ in detail, and then summarize the results for $R_{2}$.

4.3.1 Drawing the diagram Figures 4.2 and 4.3 illustrate the construction of the surface diagram for the first surface, $R_{1}$. Our choice of basepoints ensures that the resulting $B$ arc of $K=\partial R_{1}$ does not intersect the $\alpha$ curves. When removing the 3 intersections of the $A$ arc with the $\beta$ curves, we use only isotopies of the $\beta$ curves. Thus the resulting surface diagram is extremal in the sense that we do not use any stabilizations in the algorithm from Section 3.2.

The bottom of Figure 4.3 shows the surface diagram, ie the Heegaard diagram for the sutured manifold $S_{2}^{3}(K)$ (the knot complement with 2 parallel meridional sutures) with $R_{1}$ appearing as a quasipolygon.

At this point, it is straightforward to construct the sutured Heegaard diagram for $S^{3}\left(R_{1}\right)$. Simply remove the quasipolygon representing $R_{1}$ from $\Sigma$ (the shaded region labeled $P$ in Figure 4.3), and glue two copies of it to what remains in such a way that the gluing is along $A$ arcs on one copy and $B$ arcs on the other. This is shown in Figure 4.4

After the decomposition, there are no intersection points of $\alpha$ and $\beta$ curves lying on the two quasipolygons $P_{A}$ and $P_{B}$ which we glued to $\Sigma \backslash P$. Thus, the 3 -tuples of intersection points which comprise generators for the chain complex will be contained in $\Sigma \backslash P$. For that reason, it is convenient to erase all the $\beta$ arcs which intersect $P$ in the surface diagram of Figure 4.3. The resulting diagram is shown in Figure 4.5. This latter diagram is simpler to work with, in general, and contains the homotopy theoretic data necessary to understand the chain complex as a relatively $H_{1}\left(S^{3} \backslash R_{1}\right)$ graded group.

\subsubsection{The generators and their relative gradings From Figure 4.5, we see that} there are 10 generators for the sutured Floer chain complex. We can label these generators by triples, where $\boldsymbol{x}=x_{1} x_{2} x_{3}$ denotes the generator which contains the point labeled $x_{i}$ on $\alpha_{i}$. Given generators $\boldsymbol{x}$ and $\boldsymbol{y}$, we wish to calculate the difference of their associated Spin ${ }^{c}$-structures, $\mathfrak{s}(\boldsymbol{x})-\mathfrak{s}(\boldsymbol{y})$. To do this, join each $x_{i}$ to $y_{i}$ by an oriented arc along $\alpha_{i}$, and then join $y_{i}$ to some $x_{j}$ by an oriented arc along a $\beta$ curve. The result is a collection of closed curves $\gamma_{\boldsymbol{x}, \boldsymbol{y}}$ whose homology class we denote 
by $\epsilon(\boldsymbol{x}, \boldsymbol{y})=\left[\gamma_{\boldsymbol{x}}, \boldsymbol{y}\right] \in H_{1}\left(S^{3} \backslash N\left(R_{1}\right) ; \mathbb{Z}\right) \cong H_{1}\left(S^{3} \backslash R_{1} ; \mathbb{Z}\right)$, and refer to it as the difference class associated to $\boldsymbol{x}$ and $\boldsymbol{y}$. According to Lemma 2.17, the class $\epsilon(\boldsymbol{x}, \boldsymbol{y})$ is Poincaré dual to $\mathfrak{s}(\boldsymbol{x})-\mathfrak{s}(\boldsymbol{y}) \in H^{2}\left(S^{3} \backslash N\left(R_{1}\right), \partial ; \mathbb{Z}\right)$. Note that since the $\beta$ arcs in Figure 4.5 are connected, $\gamma_{\boldsymbol{x}}, \boldsymbol{y}$ can be taken to lie entirely in that figure. A cycle representing the difference of $\boldsymbol{x}=512$ and $\boldsymbol{y}=313$ is shown in Figure 4.5.
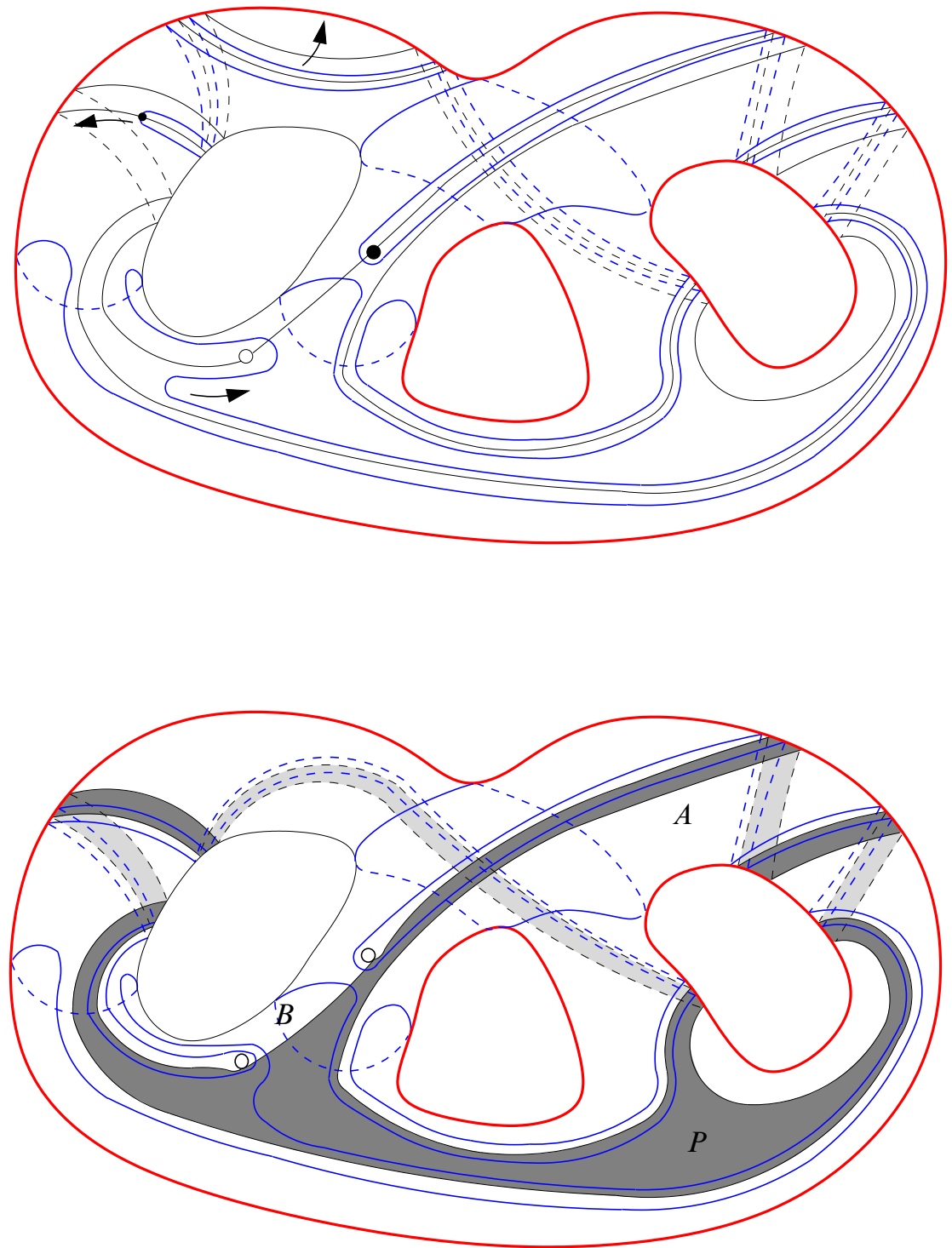

Figure 4.3: The bottom figure is the surface diagram for $R_{1}$. We have shaded the quasipolygon which represents $R_{1} \subset S^{3} \backslash K$. 
Ultimately, we wish to evaluate the Seifert form on the difference classes. To this end, it will be convenient to express $\epsilon(\boldsymbol{x}, \boldsymbol{y})$ in terms of the basis for $H_{1}\left(S^{3} \backslash R_{1}\right.$; $\left.\mathbb{Z}\right)$ given by $c_{1}, c_{2}$ in Figure 4.1. In order to do this, push the parts $\gamma_{\boldsymbol{x}, \boldsymbol{y}}$ which lie on the $\alpha$ arcs towards the $\boldsymbol{\alpha}$ handlebody (ie outwards). Similarly, push the parts of $\gamma_{\boldsymbol{x}, \boldsymbol{y}}$ lying on the $\beta$ curves towards the $\boldsymbol{\beta}$ handlebody (ie inwards). The result is a closed curve $\tilde{\gamma}_{\boldsymbol{x}}, \boldsymbol{y}$ which punctures the Heegaard surface only at the intersection points comprising $\boldsymbol{x}$ and $\boldsymbol{y}$. See Figure 4.7. Note, however, that the Heegaard surface contains the presentation of the Seifert surface in Figure 4.1. Furthermore, $\tilde{\gamma} \boldsymbol{x}_{\boldsymbol{x}} \boldsymbol{y}$ is in the complement of this presentation since the intersection points comprising $\boldsymbol{x}$ and $\boldsymbol{y}$ do not intersect the Seifert surface.

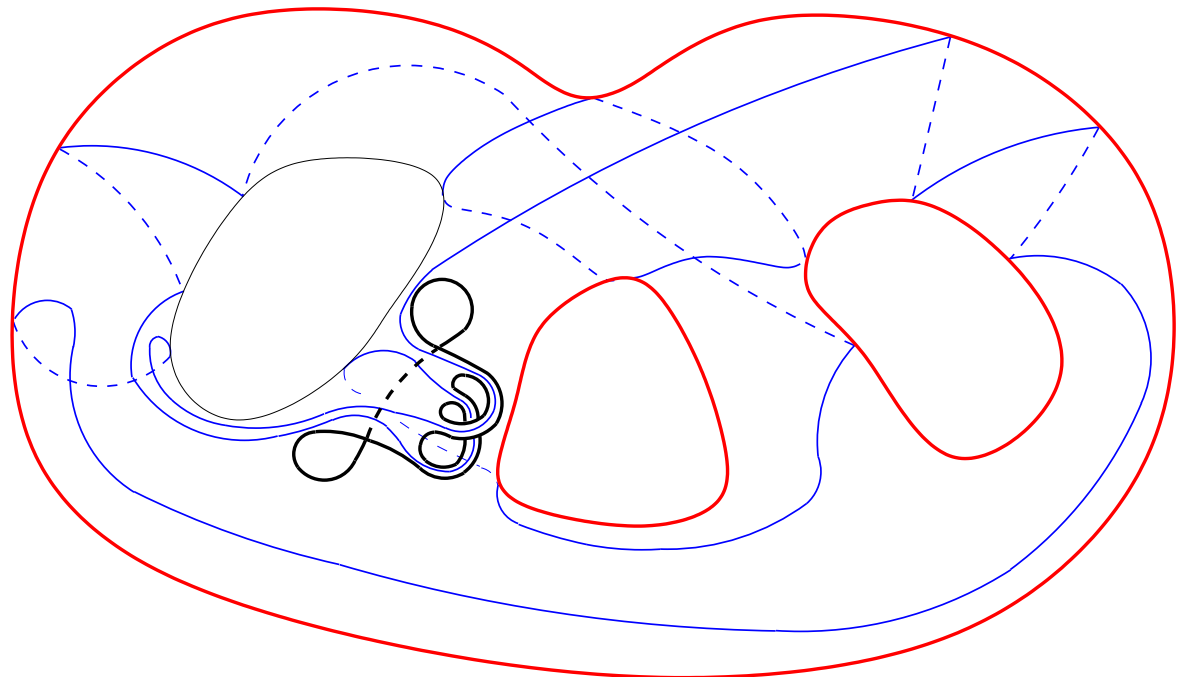

Figure 4.4: The decomposed diagram, ie the sutured diagram for $S^{3}\left(R_{1}\right)$. The bold curve is the boundary of the Heegaard surface.

Thus we can regard $\tilde{\gamma}_{\boldsymbol{x}}, \boldsymbol{y}$ in two ways: as a curve in the sutured manifold $S^{3}\left(R_{1}\right)$ presented by the decomposed diagram or as a curve in the complement of $R_{1}$, as shown in Figure 4.1. We claim that the homology class which $\tilde{\gamma}_{\boldsymbol{x}}, \boldsymbol{y}$ represents in $H_{1}\left(S^{3} \backslash R_{1}\right)$ is the same, regardless of which way we view it. In this way, we can determine the difference classes in terms of the basis for $H_{1}\left(S^{3} \backslash R_{1}\right)$ given by $c_{1}, c_{2}$ in Figure 4.1.

To prove the claim, one need only trace through the construction of the sutured diagram, starting from the presentation of $R_{1}$ in Figure 4.1. We began by constructing a diagram for $S^{3}$ which contained $R_{1}$ as a subsurface (the top part of Figure 4.2). Regarding $\tilde{\gamma}_{\boldsymbol{x}}, \boldsymbol{y}$ on this diagram, its homology class clearly agrees with that obtained by thinking of it as a curve in Figure 4.1. Indeed, removing $\beta_{1}$ and $\beta_{2}$ from this 


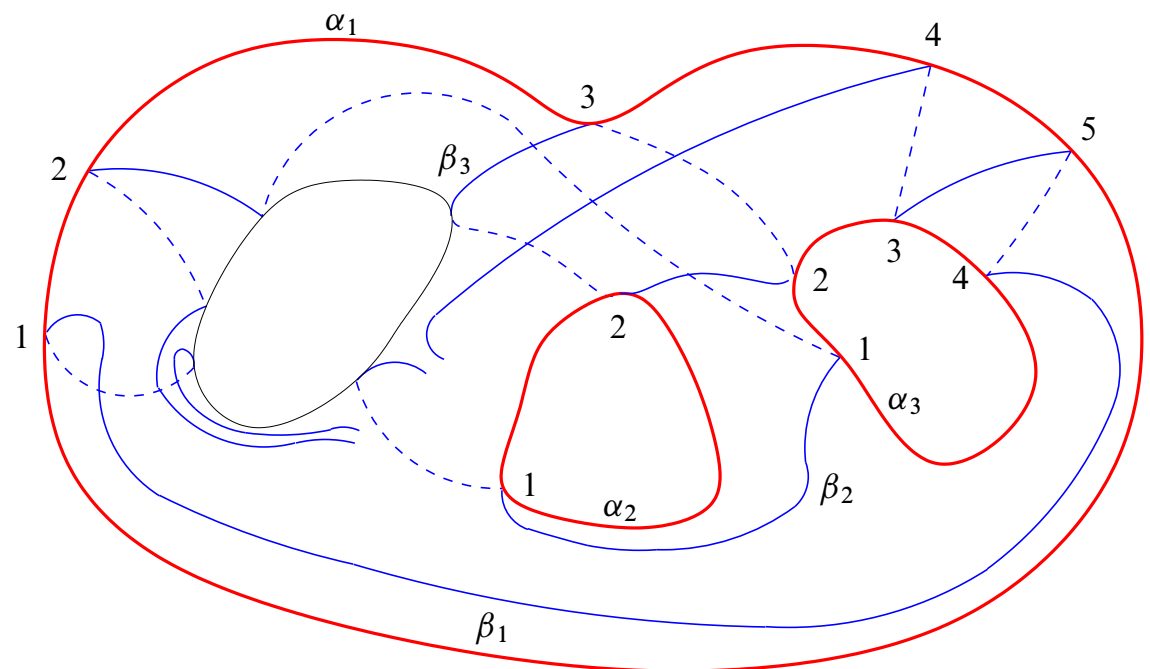

Figure 4.5: The surface diagram from Figure 4.3, where we have erased all arcs of intersection $\beta \cap P$ of the $\beta$ curves with the quasipolygon $P$ representing $R_{1}$. This diagram contains all 3-tuples of intersection points $\alpha_{i} \cap \beta_{\sigma(i)}$ which comprise the generators of $\operatorname{SFH}\left(S^{3}\left(R_{1}\right)\right)$.

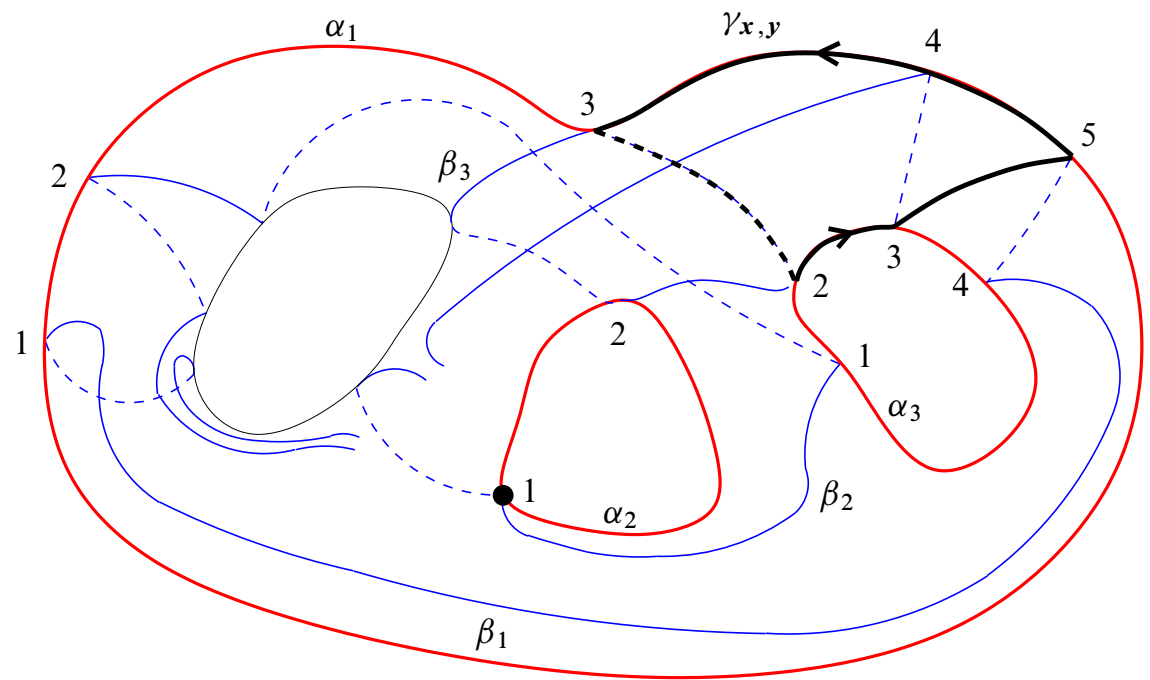

Figure 4.6: Construction of a cycle $\gamma$ representing the difference $\epsilon(\boldsymbol{x}, \boldsymbol{y})=$ $\operatorname{PD}[\mathfrak{s}(\boldsymbol{x})-\mathfrak{s}(\boldsymbol{y})] \in H_{1}\left(S^{3} \backslash R_{1}\right)$. Here $\boldsymbol{x}=512, \boldsymbol{y}=313$. The representative is comprised of two curves, one of which is constant at $1 \in \alpha_{2} \cap \beta_{2}$. 
diagram specifies $S^{3} \backslash R_{1}$ as it is presented in Figure 4.1 (see Remark 3.1). It follows that isotopies of $\beta_{1}, \beta_{2}$ do not change the homology class of $\tilde{\gamma}_{\boldsymbol{x}, \boldsymbol{y}}$ in $S^{3} \backslash R_{1}$. The surface diagram differs from the diagram for $S^{3}$ containing $R_{1}$ only by a sequence of isotopies, followed by the removal of two disks to turn it into a sutured diagram for $S_{2}^{3}(K)$. This latter modification, however, is performed far from $\tilde{\gamma}_{\boldsymbol{x}, \boldsymbol{y}}$ and hence does not effect its homology class. Another way to see this is that the surface diagram, by definition, specifies an embedding of the Seifert surface in the knot complement $S^{3} \backslash K$. From its construction, this embedding differs from the embedding shown in Figure 4.1 only by an isotopy supported in an arbitrarily small neighborhood of $\partial R_{1}$. Thus, the homology class of $\tilde{\gamma}_{\boldsymbol{x}}, \boldsymbol{y}$ in $H_{1}\left(S^{3} \backslash R_{1}\right)$ specified by the surface diagram agrees with that of Figure 4.1. Finally, decomposing the diagram is exactly the same as decomposing $S_{2}^{3}(K)$. Since $\tilde{\gamma}_{\boldsymbol{x}, \boldsymbol{y}}$ is in the complement of the quasipolygon, its homology class in $H_{1}\left(S^{3} \backslash R_{1}\right)$ (as specified by the Heegaard diagram) is unchanged by the decomposition. This proves the claim.

Figures 4.5 and 4.7 indicate that the difference class between $\boldsymbol{x}=512$ and $\boldsymbol{y}=313$ is $c_{1}$. The remaining differences are easily computed, and the following diagram represents the chain complex as a relatively $H_{1}\left(S^{3} \backslash R_{1}\right)$ graded group. (The explanation for the diagram is that each $\boldsymbol{x}$ is placed on a lattice point in the affine lattice generated by $c_{1}, c_{2}$. The difference between the lattice coordinates of $\boldsymbol{x}$ and $\boldsymbol{y}$ is the difference class $\epsilon(\boldsymbol{x}, \boldsymbol{y})$.)

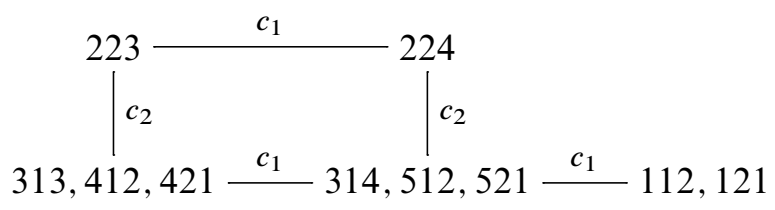

4.3.3 The homology We take our chain complexes with $\mathbb{Z} / 2 \mathbb{Z}$ coefficients. Our first observation is that the rank of the homology of the chain complex above is 4 . This follows from Theorem 2.20 above, together with the fact that $\mathrm{rk} \widehat{H F K}(K, 1)=4$. This latter fact can be seen in many ways and follows, for instance from the fact that $8_{3}$ is an alternating knot of genus 1 for which the top coefficient of the Alexander polynomial equals -4 . (According to [23, Theorem 1.3], for alternating knots, one has

$$
\text { rk } \widehat{H F K}(K, i)=\left|a_{i}\right|,
$$

where $a_{i}$ is the $i$-th coefficient of the symmetrized Alexander polynomial $\Delta_{K}(T)=$ $a_{0}+\sum_{i} a_{i}\left(T^{i}+T^{-i}\right)$.) Now the homology of each of the 2 subcomplexes in the top row of the diagram is $\mathbb{Z} / 2 \mathbb{Z}$; indeed, each complex has a single generator. This takes care of a 2-dimensional subspace of the 4-dimensional homology. As for the rest of 


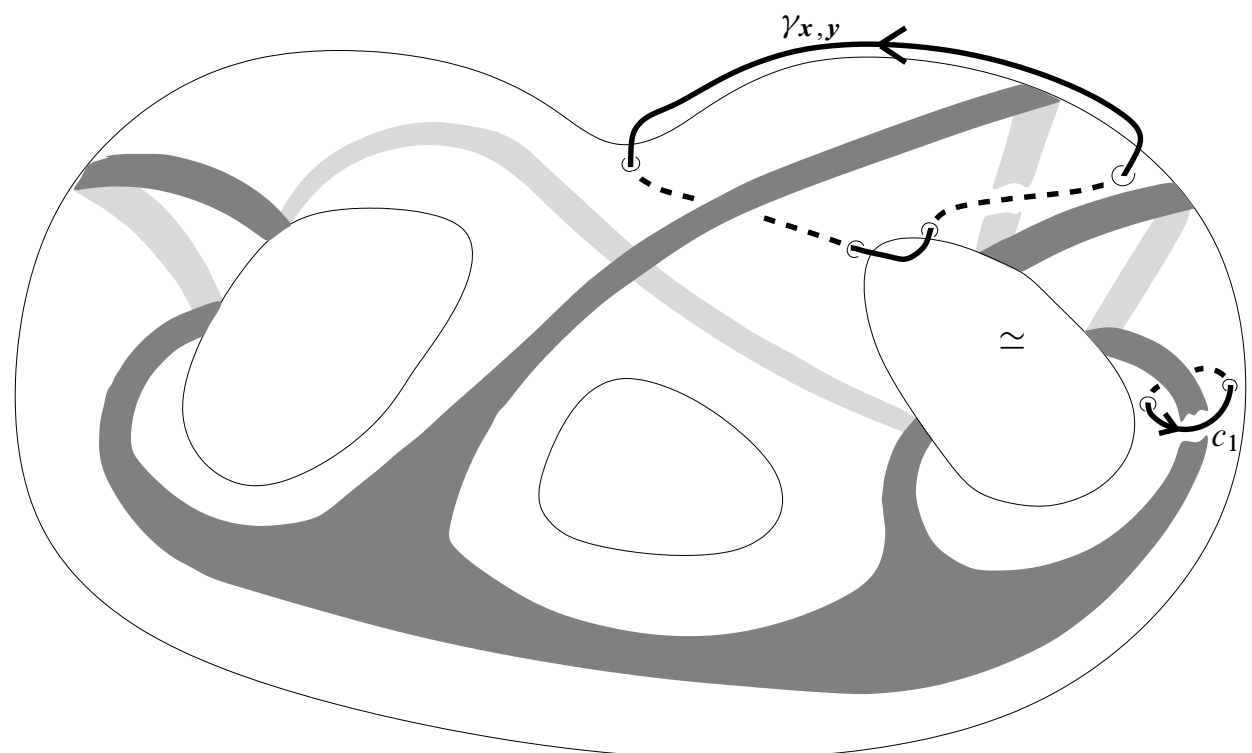

Figure 4.7: The push-off of $\gamma_{\boldsymbol{x}, \boldsymbol{y}}$ into the handlebodies yields a curve $\tilde{\gamma}_{\boldsymbol{x}, \boldsymbol{y}}$ living in the complement of $R_{1}$. In this example, $\tilde{\gamma}_{\boldsymbol{x}, \boldsymbol{y}}$ is homologous to $c_{1}$.

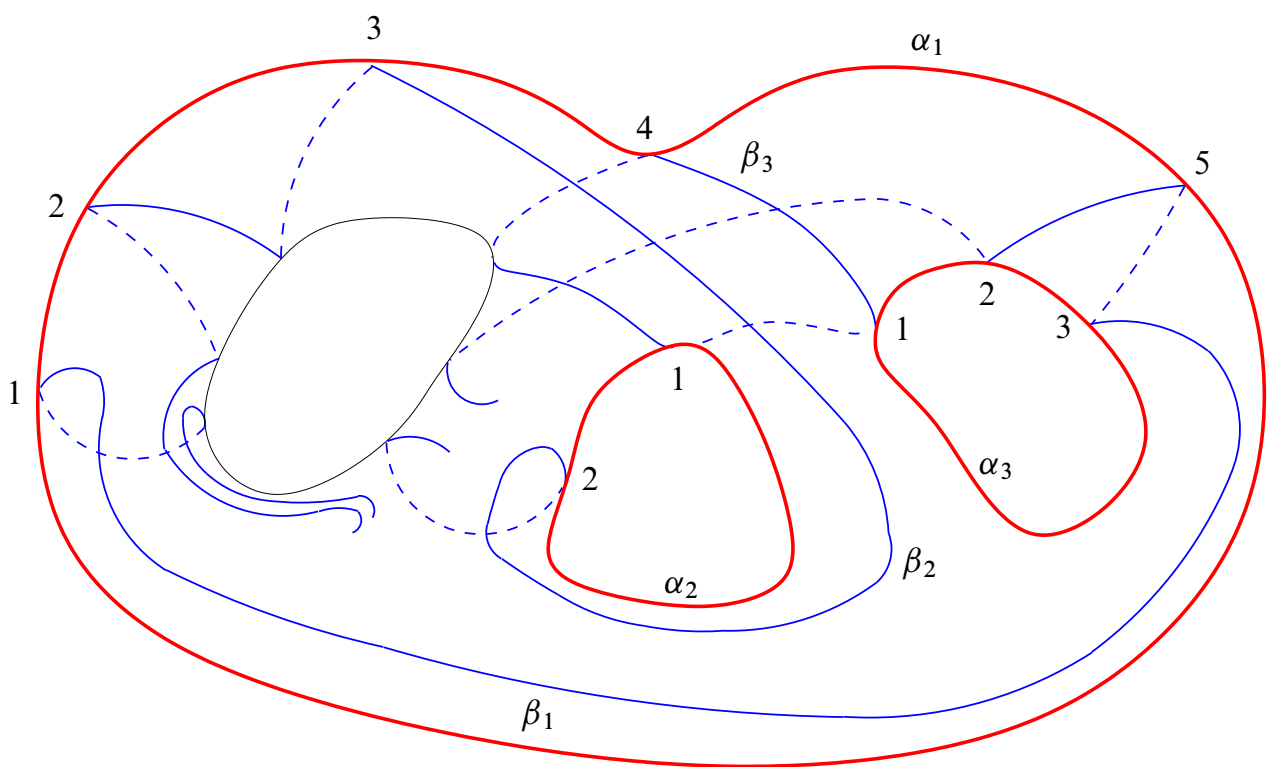

Figure 4.8: The diagram for $R_{2}$ 
the homology, note that the subcomplex generated by 112, 121 must have even Euler characteristic while the subcomplexes generated by 314, 512, 521 and 313,412,421, respectively have odd (in particular, nonzero) Euler characteristics. The only way for this to happen is if the 112,121 subcomplex is acyclic and the homology of the remaining two subcomplexes is $\mathbb{Z} / 2 \mathbb{Z}$. Summarizing, the sutured Floer homology as a relatively $H_{1}\left(S^{3} \backslash R_{1}\right)$ graded group is:

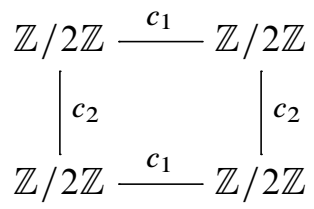

4.3.4 The results for $\boldsymbol{R}_{2}$ Figure 4.7 shows the Heegaard diagram for $R_{2}$. The resulting chain complex has 8 generators, whose relative gradings are given in terms of the basis $d_{1}, d_{2}$ of Figure 4.1 as follows:

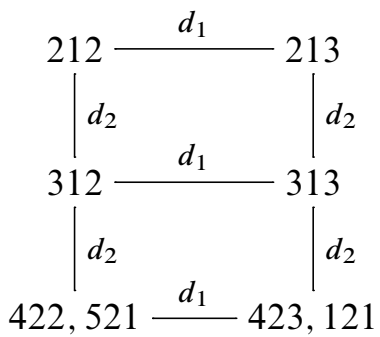

As above, the rank of the sutured Floer homology for $S^{3}\left(R_{2}\right)$ is 4 . Since the Euler characteristic of the subcomplexes generated by 422,521 and 423,121 are even, the sutured Floer homology is given by:

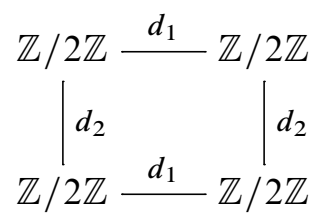

4.3.5 Distinguishing the groups Having calculated the sutured Floer homology of $S^{3}\left(R_{1}\right)$ and $S^{3}\left(R_{2}\right)$ as relatively graded groups, it remains to distinguish them.

We first remind the reader what it means for two collections of groups graded by relative $\operatorname{Spin}^{c}$-structures to be isomorphic. 
Definition 4.1 Two relative $\operatorname{Spin}^{c}$-graded groups

$$
\begin{aligned}
& \operatorname{SFH}\left(M_{1}, \gamma_{1}\right)=\bigoplus_{\mathfrak{s} \in \operatorname{Spin}^{c}\left(M_{1}, \gamma_{1}\right)} \operatorname{SFH}\left(M_{1}, \gamma_{1}, \mathfrak{s}\right), \\
& \operatorname{SFH}\left(M_{2}, \gamma_{2}\right)=\bigoplus_{\mathfrak{s} \in \operatorname{Spin}^{c}\left(M_{2}, \gamma_{2}\right)} \operatorname{SFH}\left(M_{2}, \gamma_{2}, \mathfrak{s}\right),
\end{aligned}
$$

are isomorphic (which we denote $\operatorname{SFH}\left(M_{1}, \gamma_{1}\right) \cong \operatorname{SFH}\left(M_{2}, \gamma_{2}\right)$ ) if

(1) there is an isomorphism $f^{*}: H^{2}\left(M_{1}, \partial M_{1}\right) \rightarrow H^{2}\left(M_{2}, \partial M_{2}\right)$ and a bijection $\sigma: \operatorname{Spin}^{c}\left(M_{1}, \gamma_{1}\right) \rightarrow \operatorname{Spin}^{c}\left(M_{2}, \gamma_{2}\right)$ such that the diagram

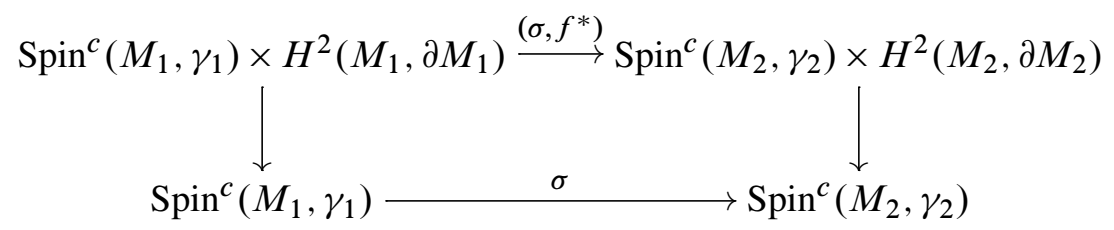

commutes, where the vertical arrows are induced by the action of $H^{2}\left(M_{i}, \partial M_{i}\right)$ on $\operatorname{Spin}^{c}\left(M_{i}, \gamma_{i}\right)$; and

(2) there are isomorphisms $g_{\mathfrak{s}}: \operatorname{SFH}\left(M_{1}, \gamma_{1}, \mathfrak{s}\right) \rightarrow \operatorname{SFH}\left(M_{2}, \gamma_{2}, \sigma(\mathfrak{s})\right)$ for every $\mathfrak{s} \in \operatorname{Spin}^{c}\left(M_{1}, \gamma_{1}\right)$.

If $f:\left(M_{2}, \gamma_{2}\right) \rightarrow\left(M_{1}, \gamma_{1}\right)$ is an equivalence, then Theorem 2.16 indicates that $\operatorname{SFH}\left(M_{1}, \gamma_{1}\right) \cong \operatorname{SFH}\left(M_{2}, \gamma_{2}\right)$. In this case, $f^{*}$ and $\sigma$ in Definition 4.1 are both obtained by pull-back along $f$. In addition, if $f$ comes from the restriction of an equivalence of Seifert surfaces $\left(S^{3}, R_{2}\right) \rightarrow\left(S^{3}, R_{1}\right)$, then $f_{*}: H_{1}\left(S^{3} \backslash R_{1}\right) \rightarrow H_{1}\left(S^{3} \backslash R_{2}\right)$ preserves the Seifert form discussed in Section 4.2, ie $a \cdot b=f_{*}(a) \cdot f_{*}(b)$.

Returning to our example, this can be made concrete as follows. Let us denote generators for the 4 nonzero Floer homology groups of $R_{1}$ (respectively $R_{2}$ ) by $\boldsymbol{x}_{i}$ (respectively $\boldsymbol{y}_{i}$ ), so that the Floer homology groups are given by
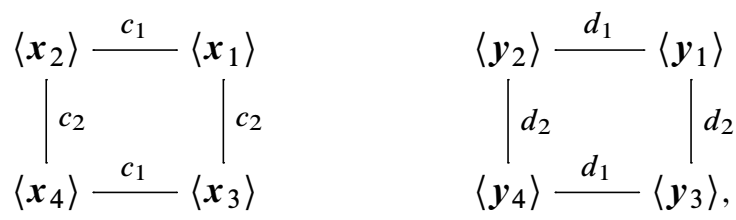

where $\langle-\rangle$ means the $\mathbb{Z} / 2 \mathbb{Z}$-vector space generated by - . Then, in order for $\operatorname{SFH}\left(S^{3}\left(R_{1}\right)\right)$ to be isomorphic to $\operatorname{SFH}\left(S^{3}\left(R_{2}\right)\right)$, there must be a bijection

$$
\sigma:\left\{x_{1}, x_{2}, x_{3}, x_{4}\right\} \longrightarrow\left\{y_{1}, y_{2}, y_{3}, y_{4}\right\},
$$


which is compatible with taking difference classes, ie $\epsilon\left(\sigma\left(\boldsymbol{x}_{i}\right), \sigma\left(\boldsymbol{x}_{j}\right)\right)=f_{*} \epsilon\left(\boldsymbol{x}_{i}, \boldsymbol{x}_{j}\right)$ for some isomorphism $f_{*}: H_{1}\left(S^{3} \backslash R_{1}\right) \rightarrow H_{1}\left(S^{3} \backslash R_{2}\right)$. Since we assume that $R_{1}$ is equivalent to $R_{2}$, the map $f_{*}$ must also preserve the Seifert form.

Suppose that such a $\sigma$ exists. Then we have

$$
\epsilon\left(\sigma\left(x_{1}\right), \sigma\left(x_{2}\right)\right)^{2}=f_{*} \epsilon\left(x_{1}, x_{2}\right)^{2}=\epsilon\left(x_{1}, x_{2}\right)^{2}=c_{1}^{2}=2,
$$

where squares indicate the pairing, under the Seifert form, of a class with itself. Thus the difference of $\sigma\left(\boldsymbol{x}_{1}\right)$ and $\sigma\left(\boldsymbol{x}_{2}\right)$ is a class whose square is 2. Considering $\epsilon\left(\boldsymbol{y}_{i}, \boldsymbol{y}_{j}\right)$ for every $i \neq j$, we obtain 8 distinct classes

$$
\pm d_{1} \quad \pm d_{2} \quad \pm\left(d_{1}+d_{2}\right) \quad \pm\left(d_{1}-d_{2}\right),
$$

whose squares are

$$
2-2 \quad-1 \quad 1,
$$

respectively. This shows that $\epsilon\left(\sigma\left(x_{1}\right), \sigma\left(x_{2}\right)\right)= \pm d_{1}$. Similarly, the fact that

$$
\epsilon\left(\sigma\left(x_{1}\right), \sigma\left(x_{3}\right)\right)^{2}=f_{*} \epsilon\left(x_{1}, x_{3}\right)^{2}=\epsilon\left(x_{1}, x_{3}\right)^{2}=c_{2}^{2}=-2
$$

implies that $\epsilon\left(\sigma\left(\boldsymbol{x}_{1}\right), \sigma\left(\boldsymbol{x}_{2}\right)\right)= \pm d_{2}$. We have arrived at a contradiction. For on the one hand

$$
\epsilon\left(\sigma\left(x_{1}\right), \sigma\left(x_{2}\right)\right) \cdot \epsilon\left(\sigma\left(x_{1}\right), \sigma\left(x_{3}\right)\right)=\epsilon\left(x_{1}, x_{2}\right) \cdot \epsilon\left(x_{1}, x_{3}\right)=c_{1} \cdot c_{2}=0,
$$

while on the other we have shown

$$
\epsilon\left(\sigma\left(x_{1}\right), \sigma\left(x_{2}\right)\right) \cdot \epsilon\left(\sigma\left(x_{1}\right), \sigma\left(x_{3}\right)\right)= \pm d_{1} \cdot \pm d_{2},
$$

and this latter pairing is nonzero, regardless of signs. This shows that $\operatorname{SFH}\left(S^{3}\left(R_{1}\right)\right) \not$ $\operatorname{SFH}\left(S^{3}\left(R_{2}\right)\right)$, and hence $R_{1} \not 2 R_{2}$.

Remark 4.2 Our argument shows that there does not exist an orientation-preserving diffeomorphism of $S^{3}$ which takes $R_{1}$ to $R_{2}$. There is, however, an obvious orientationreversing diffeomorphism which sends $R_{1}$ to $R_{2}$. To see this, simply reflect $R_{1}$ across the plane of the page, then rotate $180^{\circ}$ around a vertical axis through the middle of the surface. The composition of the reflection and rotation is the aforementioned diffeomorphism. Our result, then, can be interpreted as saying that sutured Floer homology detects "chirality" of Seifert surfaces. On the other hand, it is an interesting fact that the knot $8_{3}$ is fully amphichiral. 


\subsection{A few consequences}

Proposition 4.3 $R_{1}$ and $R_{2}$ represent all equivalence classes of minimal genus Seifert surfaces for $8_{3}$.

Proof By [15] the knot $8_{3}$ has exactly two Seifert surfaces up to strong equivalence, namely $R_{1}$ and $R_{2}$. We have just seen above that these two surfaces are inequivalent. The result follows.

Theorem 4.4 For any $n \geq 1$, there exists a knot $K_{n}$ with free Seifert surfaces $\left\{F_{0}, \ldots F_{n}\right\}$, such that $F_{i}$ is not equivalent to $F_{j}$ for any $i \neq j$.

Proof Take $K_{n}$ to the connected sum of $n$ copies of $8_{3}$, and let $F_{i}$ be the Seifert surface obtained by forming the boundary connected sum of $i$ copies of $R_{1}$ and $n-i$ and copies of the $R_{2}$. Note that we can perform $n-1$ product disk decompositions ${ }^{3}$ to $S^{3}\left(F_{i}\right)$ to obtain a sutured manifold which is equivalent to the disjoint union of $i$ copies of $S^{3}\left(R_{1}\right)$ and $n-i$ copies of $S^{3}\left(R_{2}\right)$. Now sutured Floer homology is unchanged under product decompositions (see [11, Lemma 9.13]), and under disjoint union behaves according to the Künneth principle:

$$
\operatorname{SFH}\left(Y_{1} \sqcup Y_{2}, \gamma_{1} \sqcup \gamma_{2}, \mathfrak{s}_{1} \sqcup \mathfrak{s}_{2}\right) \cong \operatorname{SFH}\left(Y_{1}, \gamma_{1}, \mathfrak{s}_{1}\right) \otimes \operatorname{SFH}\left(Y_{2}, \gamma_{2}, \mathfrak{s}_{2}\right)
$$

(where we work with $\mathbb{Z} / 2 \mathbb{Z}$ coefficients to avoid any Tor terms). Using these facts together with the calculation from the previous section, we can distinguish the number of copies of $R_{1}$ used to form $F_{i}$ as follows. First, observe that $\operatorname{rk} \operatorname{SFH}\left(S^{3}\left(F_{i}\right)\right)=4^{n}$. A generating set for the Floer homology of $F_{i}$ is given by $n$-tuples $\boldsymbol{x}_{\boldsymbol{j}} \boldsymbol{y}_{\boldsymbol{m}}$, with $\boldsymbol{j}=\left\{j_{1}, \ldots, j_{i}\right\}, \boldsymbol{m}=\left\{m_{1}, \ldots m_{n-i}\right\}$ and $j_{l}, m_{k} \in\{1,2,3,4\}$. The $n$-tuple $\boldsymbol{x}_{\boldsymbol{j}} \boldsymbol{y}_{\boldsymbol{m}}$ corresponds to

$$
\boldsymbol{x}_{j_{1}}^{1} \otimes \cdots \otimes \boldsymbol{x}_{j_{i}}^{i} \otimes \boldsymbol{y}_{m_{1}}^{1} \otimes \cdots \otimes y_{m_{n-i}}^{n-i},
$$

where $\boldsymbol{x}_{j_{l}}^{l}$ (respectively $\boldsymbol{y}_{j_{l}}^{l}$ ) is one of the 4 generators of the sutured Floer homology of the $l$-th copy of $R_{1}$ (respectively $R_{2}$ ) used to form $F_{i}$. The difference classes associated to the generators are then given by $\epsilon\left(\boldsymbol{x}_{\boldsymbol{j}} \boldsymbol{y}_{\boldsymbol{m}}, \boldsymbol{x}_{\boldsymbol{j}^{\prime}} \boldsymbol{y}_{\boldsymbol{m}^{\prime}}\right)=\epsilon\left(\boldsymbol{x}_{j_{1}}, \boldsymbol{x}_{j_{1}^{\prime}}\right) \oplus \cdots \oplus \epsilon\left(\boldsymbol{x}_{j_{i}}, \boldsymbol{x}_{j_{i}^{\prime}}\right) \oplus \epsilon\left(\boldsymbol{y}_{m_{1}}, \boldsymbol{x}_{m_{1}^{\prime}}\right) \oplus \cdots \oplus \epsilon\left(\boldsymbol{y}_{m_{1}}, \boldsymbol{y}_{m_{1}^{\prime}}\right)$, with $\epsilon\left(\boldsymbol{x}_{j_{l}}, \boldsymbol{x}_{j_{l}^{\prime}}\right)$, respectively $\epsilon\left(\boldsymbol{y}_{j_{l}}, \boldsymbol{y}_{j_{l}^{\prime}}\right)$, being one of the 8 distinct differences

$$
\pm c_{1}^{l}, \quad \pm c_{2}^{l}, \quad \pm\left(c_{1}^{l}+c_{2}^{l}\right), \quad \pm\left(c_{1}^{l}-c_{2}^{l}\right),
$$

${ }^{3} \mathrm{~A}$ product disk decomposition is a surface decomposition along a properly embedded disk which intersects the sutures in exactly 2 points. 
respectively

$$
\pm d_{1}^{l}, \quad \pm d_{2}^{l}, \quad \pm\left(d_{1}^{l}+d_{2}^{l}\right), \quad \pm\left(d_{1}^{l}-d_{2}^{l}\right) .
$$

Here, as throughout, the upper indices on $c_{k}^{l}$ (respectively $d_{k}^{l}$ ) are used to denote an element in $H_{1}\left(S^{3} \backslash F_{i}\right)$ which comes from the $l$-th copy of $R_{1}$ (respectively $R_{2}$ ). Finally, we note that the Seifert form on $H_{1}\left(S^{3}\left(F_{i}\right)\right) \cong \mathbb{Z}^{2 n}$ splits as a sum,

$$
Q_{F_{i}}=Q_{R_{1}^{1}} \oplus \cdots \oplus Q_{R_{1}^{i}} \oplus Q_{R_{2}^{1}} \oplus \cdots \oplus Q_{R_{2}^{n-i}}
$$

Now suppose that $F_{i}$ is isotopic to $F_{k}$ for some $i \neq k$. As in the previous section, this implies that there is a bijection between generators

$$
\sigma:\left\{\boldsymbol{x}_{\boldsymbol{j}} \boldsymbol{y}_{\boldsymbol{m}}\right\} \longrightarrow\left\{\tilde{\boldsymbol{x}}_{\boldsymbol{j}} \tilde{\boldsymbol{y}} \boldsymbol{m}_{\boldsymbol{m}}\right\},
$$

which is compatible with an isomorphism $f_{*}: H_{1}\left(S^{3} \backslash F_{i}\right) \rightarrow H_{1}\left(S^{3} \backslash F_{k}\right)$ which preserves the Seifert form. We use $\sim$ to distinguish generators for $F_{k}$ from those for $F_{i}$. Abusing notation, for $k \in \mathbb{Z}$ let $\mathbf{k}=\{k, k, \ldots, k\}$ denote the vector of any length, all of whose entries are $k$. We have

$$
\begin{aligned}
\epsilon\left(\sigma\left(x_{1} y_{1}\right), \sigma\left(x_{2} y_{2}\right)\right)^{2} & =f_{*} \epsilon\left(x_{1} y_{1}, x_{2} y_{2}\right)^{2}=\epsilon\left(x_{1} y_{1}, x_{2} y_{2}\right)^{2} \\
& =\left(c_{1}^{1}+\cdots+c_{1}^{i}+d_{1}^{1}+\cdots+d_{1}^{n-i}\right)^{2}=2 n .
\end{aligned}
$$

It follows that

$$
\epsilon\left(\sigma\left(\boldsymbol{x}_{\mathbf{1}} \boldsymbol{y}_{\mathbf{1}}\right), \sigma\left(\boldsymbol{x}_{\mathbf{2}} \boldsymbol{y}_{\mathbf{2}}\right)\right)=\delta_{1} c_{1}^{1}+\cdots+\delta_{k} c_{1}^{k}+\rho_{1} d_{1}^{1}+\cdots+\rho_{n-k} d_{1}^{n-k},
$$

for some choice of signs $\delta_{l}, \rho_{l} \in\{-1,1\}$. Indeed, these are the only elements in $H_{1}\left(S^{3} \backslash F_{k}\right)$ of square $2 n$ which arise as differences of generators. A similar analysis shows that

$$
\epsilon\left(\sigma\left(\boldsymbol{x}_{\mathbf{1}} \boldsymbol{y}_{\mathbf{1}}\right), \sigma\left(\boldsymbol{x}_{\mathbf{3}} \boldsymbol{y}_{\mathbf{3}}\right)\right)=\delta_{1}^{\prime} c_{2}^{1}+\cdots+\delta_{k}^{\prime} c_{2}^{k}+\rho_{1}^{\prime} d_{2}^{1}+\cdots+\rho_{n-k}^{\prime} d_{2}^{n-k},
$$

as these are the only elements in $H_{1}\left(S^{3} \backslash F_{k}\right)$ of square $-2 n$. We claim that the signs must agree in both cases; that is, $\delta_{l}^{\prime}=\delta_{l}$ and $\rho_{l}^{\prime}=\rho_{l}$ for all $l$. To see this, observe that $f_{*}$, in addition to preserving the Seifert form, must preserve the intersection product on $H_{1}\left(S^{3} \backslash F_{i}\right)$ inherited from $H_{1}\left(F_{i}\right)$ (equivalently, $f_{*}$ must be a symplectomorphism of the symplectic vector space $\left.H_{1}\left(S^{3} \backslash F_{i} ; \mathbb{R}\right)\right)$. Denoting this product by $\cap$, we have

$$
\begin{aligned}
& \epsilon\left(\boldsymbol{x}_{1} \boldsymbol{y}_{1}, \boldsymbol{x}_{\mathbf{2}} \boldsymbol{y}_{\mathbf{2}}\right) \cap \epsilon\left(\boldsymbol{x}_{\mathbf{1}} \boldsymbol{y}_{\mathbf{1}}, \boldsymbol{x}_{\mathbf{3}} \boldsymbol{y}_{\mathbf{3}}\right) \\
& \quad=\left(c_{1}^{1}+\cdots+c_{1}^{i}+d_{1}^{1}+\cdots+d_{1}^{n-i}\right) \cap\left(c_{2}^{1}+\cdots+c_{2}^{i}+d_{2}^{1}+\cdots+d_{2}^{n-i}\right)=n .
\end{aligned}
$$


On the other hand,

$$
\begin{aligned}
& \epsilon\left(\sigma\left(\boldsymbol{x}_{\mathbf{1}} \boldsymbol{y}_{\mathbf{1}}\right), \sigma\left(\boldsymbol{x}_{\mathbf{2}} \boldsymbol{y}_{\mathbf{2}}\right)\right) \cap \epsilon\left(\sigma\left(\boldsymbol{x}_{\mathbf{1}} \boldsymbol{y}_{\mathbf{1}}\right), \sigma\left(\boldsymbol{x}_{\mathbf{3}} \boldsymbol{y}_{\mathbf{3}}\right)\right) \\
& =f_{*} \epsilon\left(\boldsymbol{x}_{\mathbf{1}} \boldsymbol{y}_{\mathbf{1}}, \boldsymbol{x}_{\mathbf{2}} \boldsymbol{y}_{\mathbf{2}}\right) \cap f_{*} \epsilon\left(\boldsymbol{x}_{\mathbf{1}} \boldsymbol{y}_{\mathbf{1}}, \boldsymbol{x}_{\mathbf{3}} \boldsymbol{y}_{\mathbf{3}}\right) \\
& =\left(\delta_{1} c_{1}^{1}+\cdots+\delta_{k} c_{1}^{k}+\rho_{1} d_{1}^{1}+\cdots+\rho_{n-k} d_{1}^{n-k}\right) \\
& \quad \cap\left(\delta_{1}^{\prime} c_{2}^{1}+\cdots+\delta_{k}^{\prime} c_{2}^{k}+\rho_{1}^{\prime} d_{2}^{1}+\cdots+\rho_{n-k}^{\prime} d_{2}^{n-k}\right) \\
& =\delta_{1} \delta_{1}^{\prime} c_{1}^{1} \cap c_{2}^{1}+\cdots+\delta_{k} \delta_{k}^{\prime} c_{1}^{k} \cap c_{2}^{k}+\rho_{1} \rho_{1}^{\prime} d_{1}^{1} \cap d_{2}^{1}+\cdots+\rho_{n-k} \rho_{n-k}^{\prime} d_{1}^{n-k} \cap d_{2}^{n-k} \\
& =\delta_{1} \delta_{1}^{\prime}+\cdots+\delta_{k} \delta_{k}^{\prime}+\rho_{1} \rho_{1}^{\prime}+\cdots+\rho_{n-k} \rho_{n-k}^{\prime},
\end{aligned}
$$

and this latter expression can equal $n$ only when $\delta_{l}^{\prime}=\delta_{l}$ and $\rho_{l}^{\prime}=\rho_{l}$ for all $l$. This proves the claim.

To complete the proof of the theorem, calculate $\epsilon\left(\boldsymbol{x}_{1} \boldsymbol{y}_{1}, \boldsymbol{x}_{\mathbf{2}} \boldsymbol{y}_{2}\right) \cdot \epsilon\left(\boldsymbol{x}_{\mathbf{1}} \boldsymbol{y}_{1}, \boldsymbol{x}_{\mathbf{3}} \boldsymbol{y}_{3}\right)=c_{1}^{1} \cdot c_{2}^{1}+\cdots+c_{1}^{i} \cdot c_{2}^{i}+d_{1}^{1} \cdot d_{2}^{1}+\cdots+d_{1}^{n-i} \cdot d_{2}^{n-i}=i-n$. Since $f_{*}$ preserves the Seifert form, this should be equal to

$$
\begin{aligned}
& \epsilon\left(\sigma\left(\boldsymbol{x}_{\mathbf{1}} \boldsymbol{y}_{\mathbf{1}}\right), \sigma\left(\boldsymbol{x}_{\mathbf{2}} \boldsymbol{y}_{\mathbf{2}}\right)\right) \cdot \epsilon\left(\sigma\left(\boldsymbol{x}_{\mathbf{1}} \boldsymbol{y}_{\mathbf{1}}\right), \sigma\left(\boldsymbol{x}_{\mathbf{3}} \boldsymbol{y}_{\mathbf{3}}\right)\right) \\
& \quad=\delta_{1} \delta_{1}^{\prime} c_{1}^{1} \cdot c_{2}^{1}+\cdots+\delta_{k} \delta_{k}^{\prime} c_{1}^{k} \cdot c_{2}^{k}+\rho_{1} \rho_{1}^{\prime} d_{1}^{1} \cdot d_{2}^{1}+\cdots+\rho_{n-k} \rho_{n-k}^{\prime} d_{1}^{n-k} \cdot d_{2}^{n-k} \\
& \quad=k-n .
\end{aligned}
$$

Since $i \neq k$, the proof is complete.

\section{References}

[1] W R Alford, Complements of minimal spanning surfaces of knots are not unique, Ann. of Math. 91 (1970) 419-424 MR0253312

[2] I Altman, Sutured Floer homology distinguishes between Seifert surfaces, Topology Appl. 159 (2012) 3143-3155 MR2948273

[3] J R Eisner, Knots with infinitely many minimal spanning surfaces, Trans. Amer. Math. Soc. 229 (1977) 329-349 MR0440528

[4] S Friedl, A Juhász, J Rasmussen, The decategorification of sutured Floer homology, J. Topol. 4 (2011) 431-478 MR2805998

[5] D Gabai, Foliations and genera of links, $\mathrm{PhD}$ thesis, Princeton University (1980) MR2630652 Available at http://tinyurl.com/a5teza5

[6] D Gabai, Foliations and the topology of 3-manifolds, J. Differential Geom. 18 (1983) 445-503 MR723813 
[7] D Gabai, The Murasugi sum is a natural geometric operation, from: "Low-dimensional topology”, (S J Lomonaco, Jr, editor), Contemp. Math. 20, Amer. Math. Soc. (1983) 131-143 MR718138

[8] D Gabai, Detecting fibred links in $S^{3}$, Comment. Math. Helv. 61 (1986) 519-555 MR870705

[9] D Gabai, Foliations and the topology of 3-manifolds. II, J. Differential Geom. 26 (1987) 461-478 MR910017

[10] P Ghiggini, Knot Floer homology detects genus-one fibred knots, Amer. J. Math. 130 (2008) 1151-1169 MR2450204

[11] A Juhász, Holomorphic discs and sutured manifolds, Algebr. Geom. Topol. 6 (2006) 1429-1457 MR2253454

[12] A Juhász, Floer homology and surface decompositions, Geom. Topol. 12 (2008) 299_ 350 MR2390347

[13] A Juhász, The sutured Floer homology polytope, Geom. Topol. 14 (2010) 1303-1354 MR2653728

[14] O Kakimizu, Finding disjoint incompressible spanning surfaces for a link, Hiroshima Math. J. 22 (1992) 225-236 MR1177053

[15] O Kakimizu, Classification of the incompressible spanning surfaces for prime knots of 10 or less crossings, Hiroshima Math. J. 35 (2005) 47-92 MR2131376

[16] T Kobayashi, Uniqueness of minimal genus Seifert surfaces for links, Topology Appl. 33 (1989) 265-279 MR1026928

[17] R Lipshitz, A cylindrical reformulation of Heegaard Floer homology, Geom. Topol. 10 (2006) 955-1097 MR2240908

[18] H C Lyon, Simple knots without unique minimal surfaces, Proc. Amer. Math. Soc. 43 (1974) 449-454 MR0377850

[19] C Manolescu, P Ozsváth, S Sarkar, A combinatorial description of knot Floer homology, Ann. of Math. 169 (2009) 633-660 MR2480614

[20] Y Ni, Sutured Heegaard diagrams for knots, Algebr. Geom. Topol. 6 (2006) 513-537 MR2220687

[21] Y Ni, Knot Floer homology detects fibred knots, Invent. Math. 170 (2007) 577-608 MR2357503

[22] Y Ni, Erratum: Knot Floer homology detects fibred knots, Invent. Math. 177 (2009) 235-238 MR2507641

[23] P Ozsváth, Z Szabó, Heegaard Floer homology and alternating knots, Geom. Topol. 7 (2003) 225-254 MR1988285

[24] P Ozsváth, Z Szabó, Holomorphic disks and genus bounds, Geom. Topol. 8 (2004) 311-334 MR2023281 
[25] P Ozsváth, Z Szabó, Holomorphic disks and knot invariants, Adv. Math. 186 (2004) 58-116 MR2065507

[26] P Ozsváth, Z Szabó, Holomorphic disks and three-manifold invariants: properties and applications, Ann. of Math. 159 (2004) 1159-1245 MR2113020

[27] P Ozsváth, Z Szabó, Holomorphic disks and topological invariants for closed threemanifolds, Ann. of Math. 159 (2004) 1027-1158 MR2113019

[28] J A Rasmussen, Floer homology and knot complements, PhD thesis, Harvard University (2003) MR2704683 Available at http://tinyurl.com/bearjph

[29] S Sarkar, J Wang, An algorithm for computing some Heegaard Floer homologies, Ann. of Math. 171 (2010) 1213-1236 MR2630063

[30] W P Thurston, A norm for the homology of 3-manifolds, Mem. Amer. Math. Soc. 59 (1986) i-vi and 99-130 MR823443

[31] H F Trotter, Some knots spanned by more than one knotted surface of mininal genus, from: "Knots, groups, and 3-manifolds", (L P Neuwirth, editor), Ann. of Math. Studies 84, Princeton Univ. Press (1975) 51-52 MR0377851

[32] V Turaev, Torsion invariants of $\operatorname{Spin}^{c}$-structures on 3-manifolds, Math. Res. Lett. 4 (1997) 679-695 MR1484699

Department of Mathematics, Michigan State University

East Lansing, MI 48824, USA

Department of Mathematics, South Kensington Campus, Imperial College

London SW7 2AZ, UK

Department of Mathematics, Princeton University

Princeton, NJ 08544, USA

mhedden@math.msu.edu, a.juhasz@imperial.ac.uk, sucharit@math.princeton.edu

http://www . math.msu.edu/ mhedden/Site/Home.html, http://www2.imperial.ac.uk/ ajuhasz/

Received: 9 March 2011 